\title{
PRECESSION-NUTATIONS AND TIDAL POTENTIAL
}

\author{
P. MELCHIOR
}

Observatoire Royal de Belgique, Bruxelles, Belgium

\author{
(Presented at IAU Colloquium No. 9, 'The IAU System of \\ Astronomical Constants', Heidelberg, Germany, August 12-14, 1970.)
}

\section{Basic Equations}

We have previously established (Melchior and Georis, 1968) the equations connecting the development of precession-nutation with the tesseral part of the tidal potential. These equations are:

$$
\begin{aligned}
\sin \theta \Delta \psi & =-E_{\mathbb{Q}} \sum_{i} \frac{\omega}{\Delta \omega_{i}}\left[A_{i}+A_{-i}\right] \sin \left(\Delta \omega_{i} t\right) \\
\Delta \theta & =-E_{\mathbb{Q}} \sum_{i} \frac{\omega}{\Delta \omega_{i}}\left[A_{i}-A_{-i}\right] \cos \left(\Delta \omega_{i} t\right)
\end{aligned}
$$

where

$\omega_{i}, A_{i}$ respectively represent the frequencies and amplitudes of the tidal waves, $\omega$ the Earth's rotation velocity,

$$
\begin{aligned}
\Delta \omega_{i} & =\omega_{i}-\omega \text { and } \Delta \omega_{i}=-\Delta \omega_{-i} \\
E_{\mathbb{q}} & =\frac{3}{2} \frac{f \mu}{c^{3}} \frac{C-A}{C} \frac{1}{\omega^{2}} \sim 0.0164 \quad \text { (see later). }
\end{aligned}
$$

These equations mathematically express two interesting theorems:

THEOREM I: The frequency of a nutation may directly be deduced from the frequency of the corresponding tide by simple subtraction of the 'sidereal frequency' $(15.041 / \mathrm{h}$ UT).

THEOREM II: Two waves having frequencies symmetric to the sidereal frequency form only one and the same wave of nutation; the sum of their amplitudes gives the semimajor axis and their difference the semi-minor axis of the nutation ellipse.

\section{Proposal for a Systematic Codification of Nutation Tables}

One can easily verify the non-absolutely rational construction of the IAU nutation tables. A few arguments are negative while the greatest part of them are positive; the corresponding amplitudes have been changed in sign in the $\Delta \psi$ development where the sine of these arguments appears.

Negative argument waves are marked by an $X$ in Table $I$. In addition to this, terms are listed in a completely arbitrary order, taking no account of their period. 
Doodson's tidal potential development uses Brown's tables with following arguments in the order of decreasing rate of change.

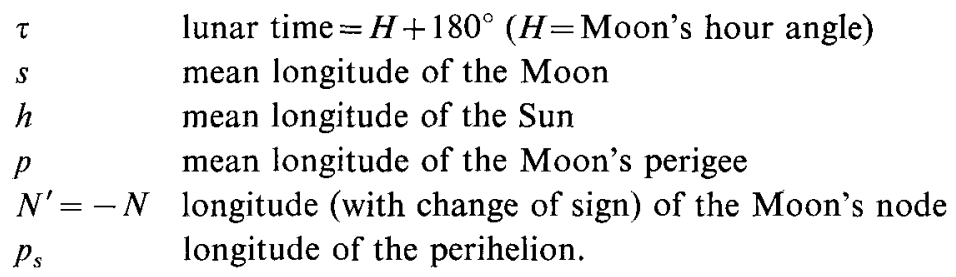

If $t$ is the mean solar time,

$$
\tau+s=t+h=T \text { sidereal } .
$$

Every tidal wave receives an argument of the form

$$
a \tau+b s+c h+d p+e N^{\prime}+f p_{s}
$$

where

$$
\begin{aligned}
& a=1 \text { for diurnal tesseral waves } \\
& -4<b, c, d, e, f<+4 \quad \text { (a few waves excepted). }
\end{aligned}
$$

Doodson introduces a very practical and efficient classification of the numberless tidal waves in associating with them a code-number noted as

$$
a, b+5, c+5 ; d+5, e+5, f+5
$$

(with $\mathrm{X}$ for 10 and $E$ for 11 ).

For example, a wave whose argument is

$$
\tau-2 s+2 h-p \text { becomes } 137.455
$$

(Table II lists the 156 principal tesseral wave arguments).

We propose to the Commission the adoption of these variables (rather than the anomalies) and the same code-number notation. The argument of a nutation can be found by subtracting the sidereal frequency $(\tau+s)$, say 110000 from the Doodson's higher argument of the couple of associated tides.

The higher tidal argument systematically should be the first of the two associated waves in Table I if astronomers did not use negative arguments for only a few waves. For all of these (X), the second tidal argument is the highest.

This kind of codification allows an automatic classification of the nutation waves in decreasing period order; the IAU Table classification is very confused as can be seen in column 'Argument of Nutation' of Table I.

The new classification authorizes a division into five groups of nutations characterized by the first code number corresponding to five period ranges (cf. Tables VA, VB).

Also, this codification allows a quite natural input of nutation tables into computers.

The code number restitutes the angular argument without any difficulty. 
Moreover this codification links together in one and the same spirit tidal and nutation theories allowing a better understanding of the fundamental link between these two problems.

\section{Comparison Between Woolard's (Nutations) and Doodson's (Tides) Developments}

Both of these authors have developed the luni-solar potential in different ways.

Woolard looks for all nutation waves whose amplitudes in $\sin \theta \Delta \psi$ or in $\Delta \theta$ are greater than 0.0002 .

Doodson seeks all tidal waves whose amplitudes $A_{i}$ are greater than $0.00010 G_{1}$ where $G_{1}$ is a geodesic coefficient:

$$
G_{1}=G \sin 2 \phi=\frac{3}{4} \mu \frac{g r^{2} a^{2}}{c^{3}} \sin 2 \phi
$$

These two limits do not absolutely correspond to the same terms because, as can be seen from (1), amplitudes $\left(A_{i}, A_{-i}\right)$ of tidal waves must be multiplied by an integration factor $\omega / \Delta \omega_{i}$ in order to give the nutation amplitudes.

Waves with a period very near to one sidereal day are much enlarged in nutation because $\Delta \omega_{i} \approx 0$.

On the other hand, non-negligible tidal waves practically disappear in nutation because their frequencies sensibly deviate from the sidereal frequency (large $\Delta \omega_{i}$ ).

Table I: This table reproduces, in the usual order, the list of IAU nutation waves. Their arguments are given in terms of Doodson's variables ( $, \mathrm{H}, \mathrm{P}, \mathrm{N}, \mathrm{PS}$, corre-

TABLE I

IAU Nutation development

\begin{tabular}{|c|c|c|c|c|c|c|c|}
\hline \multicolumn{3}{|c|}{ Argument of Nutation } & \multicolumn{2}{|c|}{ Tidal Arguments } & \multirow{2}{*}{$\begin{array}{c}\Delta \psi \\
-17.2327\end{array}$} & \multirow{2}{*}{$\begin{array}{r}\sin \theta \cdot \Delta \psi \\
-6.8584\end{array}$} & \multirow{2}{*}{$\begin{array}{l}\Delta \theta \\
9.2100\end{array}$} \\
\hline $\mathbf{N}$ & 55.565 & $X \ldots$ & 165.545 & 165.565 & & & \\
\hline $2 \mathrm{~N}$ & 55.575 & $\mathrm{X}$. & 165.535 & 165.575 & 0.2088 & 0.0831 & -0.0904 \\
\hline $2 \mathrm{H}$ & 57.555 & . & 167.555 & 163.555 & -1.2729 & -0.5066 & 0.5522 \\
\hline $\mathbf{H}-\mathrm{PS}$ & 56.554 & .. & 166.554 & 164.556 & 0.1261 & 0.0502 & $*$ \\
\hline $\mathbf{H}+\mathbf{P S}$ & 56.556 & . & 166.556 & 164.554 & 0.0214 & 0.0085 & -0.0093 \\
\hline $3 \mathrm{H}-\mathrm{PS}$ & 58.554 & .. & 168.554 & 162.556 & -0.0497 & -0.0198 & 0.0216 \\
\hline $2 \mathrm{H}-\mathrm{N}$ & 57.565 & .. & 167.565 & 163.545 & 0.0124 & 0.0049 & -0.0066 \\
\hline $2 \mathrm{H}-2 \mathrm{PS}$ & 57.553 & . & 167.553 & 163.557 & 0.0016 & 0.0006 & * \\
\hline $4 \mathrm{H}-2 \mathrm{PS}$ & 59.553 & . & 169.553 & 161.557 & -0.0015 & -0.0006 & 0.0007 \\
\hline $2 P-N$ & 55.765 & & 165.765 & 165.345 & 0.0045 & 0.0018 & -0.0024 \\
\hline $\mathbf{H}+\mathbf{N}-\mathrm{PS}$ & 56.544 & & 166.544 & 164.566 & -0.0015 & -0.0006 & 0.0008 \\
\hline$H-N-P S$ & 56.564 & - & 166.564 & 164.546 & 0.0010 & 0.0004 & 0.0005 \\
\hline $2 \mathrm{H}-2 \mathrm{P}-\mathrm{N}$ & 57.365 & & 167.365 & 163.745 & 0.0005 & 0.0002 & 0.0003 \\
\hline $\mathrm{H}-\mathrm{N}+\mathbf{P S}$ & 56.566 & & 166.566 & 164.544 & -0.0005 & -0.0002 & 0.0003 \\
\hline $2 \mathrm{PS}-\mathrm{N}$ & 55.567 & & 165.567 & 165.543 & -0.0004 & -0.0002 & 0.0002 \\
\hline $2 \mathrm{H}-2 \mathrm{P}+\mathrm{N}$ & 57.345 & & 167.345 & 163.765 & 0.0004 & 0.0002 & -0.0002 \\
\hline $3 \mathrm{H}-\mathrm{PS}-\mathrm{N}$ & 58.564 & & 168.564 & 162.546 & 0.0003 & 0.0001 & -0.0002 \\
\hline $2 \mathrm{P}$ & 55.755 & & 165.755 & 165.355 & -0.0003 & -0.0001 & 0.0002 \\
\hline $2 \mathrm{H}-2 \mathrm{P}$ & 57.355 & . & 167.355 & 163.755 & 0.0045 & 0.0018 & $*$ \\
\hline$-2 \mathrm{H}+2 \mathrm{~N}$ & 57.575 & $X \ldots$ & 163.535 & 167.575 & 0.0021 & 0.0009 & $*$ \\
\hline
\end{tabular}


Table I (Continued)

\begin{tabular}{|c|c|c|c|c|c|c|c|c|}
\hline \multicolumn{4}{|c|}{ Argument of Nutation } & \multicolumn{2}{|c|}{ Tidal Arguments } & \multirow{2}{*}{$\frac{\Delta \psi}{0.0010}$} & \multirow{2}{*}{$\frac{\sin \theta \cdot \Delta \psi}{0.0004}$} & \multirow[t]{2}{*}{$\Delta \theta$} \\
\hline$-2 \mathrm{P}+2 \mathrm{~N}$ & 55.775 & $\mathrm{X}$ & & 165.335 & 165.775 & & & \\
\hline $\mathbf{H}-\mathbf{P}$ & 56.455 & & & 166.455 & 164.655 & -0.0003 & -0.0001 & \\
\hline$\cdots \mathbf{P}+\mathbf{P S}$ & 55.654 & $\mathrm{X}$ & & 165.456 & 165.654 & -0.0002 & -0.0001 & \\
\hline $2 \mathrm{~S}$ & 75.555 & & . & 185.555 & 145.555 & -0.2037 & -0.0811 & 0.0884 \\
\hline$S-P$ & 65.455 & & . & 175.455 & 155.655 & 0.0675 & 0.0269 & * \\
\hline $2 S-N$ & 75.565 & & . & 185.565 & 145.545 & -0.0342 & -0.0136 & 0.0183 \\
\hline $3 S-P$ & 85.455 & & . & 195.455 & 135.655 & -0.0261 & -0.0104 & 0.0113 \\
\hline $\mathrm{S}+\mathrm{P}$ & 65.655 & & $\cdots$ & 175.655 & 155.455 & 0.0114 & 0.0045 & -0.0050 \\
\hline $2 \mathrm{~S}-2 \mathrm{H}$ & 73.555 & & . & 183.555 & 147.555 & 0.0060 & 0.0024 & $*$ \\
\hline$-\mathrm{S}+2 \mathrm{H}-\mathrm{P}$ & 63.655 & $\mathrm{X}$ & . & 157.455 & 173.655 & -0.0149 & -0.0059 & $*$ \\
\hline$S-P+N$ & 65.445 & & . & 175.445 & 155.665 & 0.0058 & 0.0023 & -0.0031 \\
\hline$--\mathbf{S}+\mathbf{P}+\mathbf{N}$ & 65.465 & $\mathrm{X}$ & . & 155.645 & 175.465 & -0.0057 & -0.0023 & 0.0030 \\
\hline $3 \mathrm{~S}-2 \mathrm{H}+\mathrm{P}$ & 83.655 & & $\ldots$ & 193.655 & 137.455 & -0.0052 & -0.0021 & 0.0022 \\
\hline $3 S-P-N$ & 85.465 & & . & 195.465 & 135.645 & -0.0044 & -0.0018 & 0.0023 \\
\hline $4 \mathrm{~S}-2 \mathrm{H}$ & 93.555 & & .. & $1 \times 3.555$ & 127.555 & -0.0032 & -0.0013 & 0.0014 \\
\hline $2 \mathrm{H}+\mathrm{S}-\mathrm{P}$ & 67.455 & & . & 177.455 & 153.655 & 0.0026 & 0.0010 & -0.0011 \\
\hline $4 S-2 P$ & 95.355 & & . & $1 \times 5.355$ & 125.755 & -0.0026 & -0.0010 & 0.0011 \\
\hline $\mathbf{S}+\mathrm{P}-\mathrm{N}$ & 65.665 & & . & 175.665 & 155.445 & 0.0019 & 0.0008 & -0.0010 \\
\hline$-\mathrm{S}+2 \mathrm{H}-\mathrm{P}-\mathrm{N}$ & N 63.645 & $\mathrm{X}$ & .. & 157.465 & 173.645 & -0.0014 & -0.0006 & -0.0007 \\
\hline$-\mathrm{S}+2 \mathrm{H}-\mathrm{P}+\mathrm{N}$ & ง 63.665 & $\mathrm{X}$ & . & 157.445 & 173.665 & -0.0013 & -0.0005 & 0.0007 \\
\hline $3 S-2 H-P-N$ & 83.465 & & . & 193.465 & 137.645 & -0.0009 & -0.0004 & 0.0005 \\
\hline $2 \mathbf{S}+\mathbf{H}--\mathbf{P S}$ & 76.554 & & - & 186.554 & 144.556 & 0.0007 & 0.0003 & -0.0003 \\
\hline $2 \mathrm{~S}-\mathrm{H}+\mathrm{PS}$ & 74.556 & & . & 184.556 & 146.554 & -0.0006 & -0.0002 & 0.0003 \\
\hline $5 \mathrm{~S}-2 \mathrm{H}-\mathrm{P}$ & $\times 3.455$ & & .. & $1 \mathrm{E} 3.455$ & 117.655 & -0.0006 & -0.0002 & 0.0003 \\
\hline $2 \mathrm{~S}+2 \mathrm{H}-2 \mathrm{P}$ & 77.355 & & - & 187.355 & 143.755 & 0.0006 & 0.0002 & -0.0002 \\
\hline$-2 \mathrm{~S}+2 \mathrm{H}-\mathrm{N}$ & 73.545 & $\mathrm{X}$ & . & 147.565 & 183.545 & 0.0006 & 0.0002 & 0.0003 \\
\hline $\mathrm{S}+2 \mathrm{H}-\mathrm{P}-\mathrm{N}$ & 67.465 & & . & 177.465 & 153.645 & 0.0005 & 0.0002 & -0.0003 \\
\hline$\ldots-2 \mathrm{~S}+2 \mathrm{H}+\mathrm{N}$ & 73.565 & $\mathrm{X}$ & . & 147.545 & 183.565 & -0.0005 & -0.0002 & 0.0003 \\
\hline $4 S-2 H-N$ & 93.565 & & . & $1 \times 3.565$ & 127.545 & -0.0005 & -0.0002 & 0.0003 \\
\hline $4 S-2 P-N$ & 95.365 & & . & $1 \times 5.365$ & 125.745 & -0.0004 & -0.0002 & 0.0002 \\
\hline $2 S-2 P$ & 75.355 & & . & 185.355 & 145.755 & 0.0028 & 0.0011 & * \\
\hline $2 \mathrm{~S}-2 \mathrm{~N}$ & 75.575 & & . & 185.575 & 145.535 & 0.0025 & 0.0010 & * \\
\hline$--\mathrm{S}+3 \mathrm{H}-\mathrm{P}-\mathrm{PS}$ & 62.656 & $\mathrm{X}$ & . & 158.454 & 172.656 & -0.0007 & -0.0003 & $*$ \\
\hline$-3 \mathrm{~S}+2 \mathrm{H}+\mathbf{P}$ & 83.455 & $\mathrm{X}$ & . & 137.655 & 193.455 & -0.0006 & -0.0002 & * \\
\hline$-\mathrm{S}+\mathrm{H}+\mathrm{P}-\mathrm{PS}$ & 64.456 & $\mathrm{X}$ & . & 156.654 & 174.456 & -0.0004 & -0.0002 & \\
\hline$-2 \mathrm{~S}+3 \mathrm{H}-\mathrm{PS}$ & 72.556 & $\mathrm{X}$ & $\ldots$ & 148.554 & 182.556 & -0.0004 & -0.0002 & \\
\hline$-\mathbf{S}+\mathbf{H}$ & 64.555 & $\mathrm{X}$ & .. & 156.555 & 174.555 & 0.0004 & 0.0002 & * \\
\hline$-S-P+2 N$ & 65.675 & $\mathrm{X}$ & .. & 155.435 & 175.675 & 0.0004 & 0.0002 & * \\
\hline $3 S-P-2 N$ & 85.475 & & . & 195.475 & 135.635 & 0.0003 & 0.0001 & $*$ \\
\hline $\mathbf{S}+\mathbf{H}-\mathbf{P}-\mathbf{P S}$ & 66.454 & & . & 176.454 & 154.656 & -0.0003 & -0.0001 & * \\
\hline $3 S-H-P+P S$ & 84.456 & & . & 194.456 & 136.654 & -0.0003 & -0.0001 & \\
\hline$-2 S+2 P+N$ & 75.365 & $\mathrm{X}$ & . & 145.745 & 185.365 & -0.0002 & -0.0001 & \\
\hline$-\mathrm{S}+2 \mathrm{H}+\mathrm{P}-\mathrm{N}$ & J 63.445 & $\mathrm{X}$ & . & 157.665 & 173.445 & -0.0002 & -0.0001 & \\
\hline $2 S-2 P+N$ & 75.345 & & . & 185.345 & 145.765 & 0.0002 & 0.0001 & \\
\hline $3 \mathrm{~S}-3 \mathrm{H}+\mathrm{P}+\mathrm{PS}$ & 82.656 & & . & 192.656 & 138.454 & -0.0002 & -0.0001 & \\
\hline $4 S-3 H+P S$ & 92.556 & & . & $1 \times 2.556$ & 128.554 & -0.0002 & -0.0001 & \\
\hline$S-P+2 N$ & 65.435 & & . & 175.435 & 155.675 & -0.0002 & -0.0001 & \\
\hline $3 \mathbf{S}+\mathbf{H}-\mathbf{P}-\mathrm{PS}$ & 86.454 & & . & 196.454 & 134.656 & 0.0002 & 0.0001 & \\
\hline $5 S-3 P$ & $\mathrm{X} 5.255$ & & . & $1 \mathrm{E} 5.255$ & 115.855 & -0.0002 & -0.0001 & \\
\hline
\end{tabular}

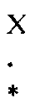

Negative argument ( $\mathrm{X}=10, \mathrm{E}=11$, if appearing in place of digits)

Component appearing in Doodson's tides development

Symmetrical waves of $K 1 /$ precession/and of equal amplitudes - no nutation in obliquity 
TABLE II

Diurnal tides - Doodson's development

$P$ Precession

N IAU nutation terms

W Woolard's nutation terms

* Symmetrical waves of $K 1 /$ precession/and of equal amplitudes - no nutation in obliquity

M Lunar waves

S Solar waves

3 Waves deriving from 3 th order potential

\begin{tabular}{cccc}
\hline Argument & Frequency & Amplitude & Remarks \\
& $\omega_{i}$ & & \\
\hline
\end{tabular}

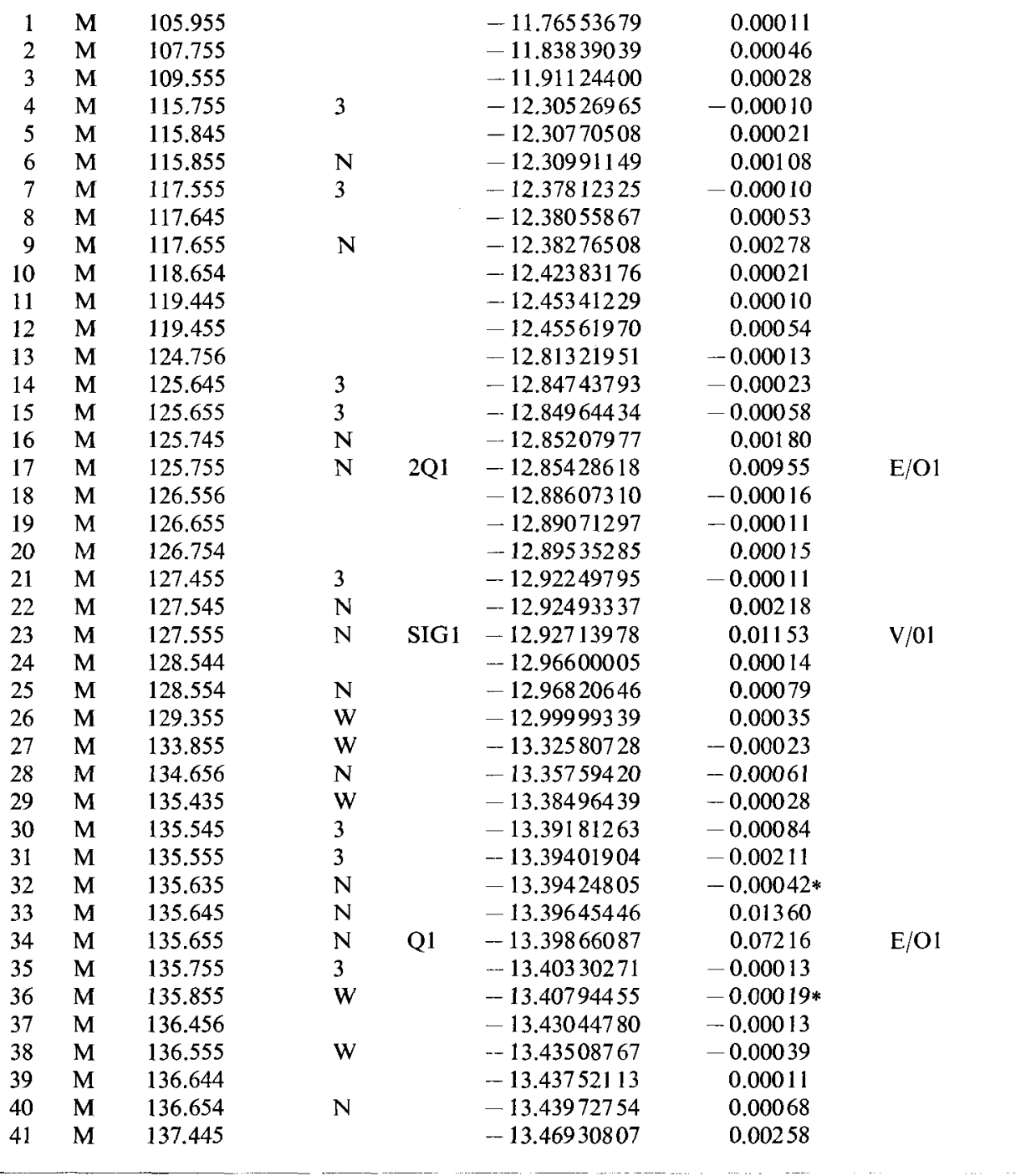


Table II (Continued)

\begin{tabular}{|c|c|c|c|c|c|c|c|}
\hline & & Argument & & & $\begin{array}{l}\text { Frequency } \\
\qquad \omega_{i}\end{array}$ & Amplitude & Remarks \\
\hline 42 & $\mathrm{M}$ & 137.455 & $\mathrm{~N}$ & RO1 & -13.47151448 & 0.01371 & $\mathrm{EV} / 01$ \\
\hline 43 & $\mathrm{M}$ & 137.555 & 3 & & -13.47615631 & -0.00018 & \\
\hline 44 & $\mathrm{M}$ & 137.655 & $\mathbf{N}$ & & -13.48079814 & $-0.00078 *$ & \\
\hline 45 & $\mathrm{M}$ & 137.665 & $\mathrm{~W}$ & & -13.48300455 & 0.00024 & \\
\hline 46 & $\mathrm{M}$ & 138.444 & & & -13.51037475 & 0.00011 & \\
\hline 47 & $\mathrm{M}$ & 138.454 & $\mathrm{~N}$ & & -13.51258116 & 0.00064 & \\
\hline 48 & $\mathbf{M}$ & 139.455 & W & & -13.55365176 & -0.00014 & \\
\hline 49 & $\mathrm{M}$ & 143.535 & W & & -13.85648548 & -0.00017 & \\
\hline 50 & $\mathrm{M}$ & 143.745 & W & & -13.86797556 & -0.00020 & \\
\hline 51 & $\mathrm{M}$ & 143.755 & $\mathrm{~N}$ & & -13.87018197 & -0.00113 & \\
\hline 52 & $\mathrm{M}$ & 144.546 & W & & -13.89976249 & -0.00015 & \\
\hline 53 & $\mathrm{M}$ & 144.556 & $\mathrm{~N}$ & & -13.90196890 & -0.00130 & \\
\hline 54 & $\mathbf{M}$ & 145.455 & 3 & & -13.93839374 & 0.00012 & \\
\hline 55 & $\mathrm{M}$ & 145.535 & $\mathrm{~N}$ & & -13.93862275 & $-0.00218 *$ & \\
\hline 56 & $\mathrm{M}$ & 145.545 & $\mathrm{~N}$ & & -13.94082916 & 0.07105 & \\
\hline 57 & $\mathrm{M}$ & 145.555 & $\mathrm{~N}$ & $\mathrm{O} 1$ & -13.94303557 & 0.37689 & \\
\hline 58 & $\mathrm{M}$ & 145.645 & 3 & & -13.94547099 & 0.00016 & \\
\hline 59 & $\mathrm{M}$ & 145.655 & 3 & & -13.94767740 & -0.00108 & \\
\hline 60 & $\mathbf{M}$ & 145.665 & 3 & & -13.94988381 & 0.00014 & \\
\hline 61 & $\mathbf{M}$ & 145.755 & $\mathbf{N}$ & & -13.95231924 & $-0.00243 *$ & \\
\hline 62 & $\mathbf{M}$ & 145.765 & $\mathrm{~N}$ & & -13.95452565 & -0.00040 & \\
\hline 63 & $\mathbf{M}$ & 146.544 & W & & -13.98189583 & 0.00012 & \\
\hline 64 & $\mathbf{M}$ & 146.554 & $\mathrm{~N}$ & & -13.98410224 & 0.00115 & \\
\hline 68 & $\mathrm{M}$ & 147.555 & $\mathrm{~N}$ & TO1 & -14.02517284 & $-0.00491 *$ & $\mathrm{~V} / \mathrm{K} 1 \mathrm{M}$ \\
\hline 65 & $\mathrm{M}$ & 147.355 & W & & -14.01588917 & -0.00021 & \\
\hline 66 & $\mathbf{M}$ & 147.455 & 3 & & -14.02053101 & -0.00021 & \\
\hline 67 & $M$ & 147.545 & $\mathrm{~N}$ & & -14.02296643 & 0.00014 & \\
\hline 69 & $\mathrm{M}$ & 147.565 & $\mathbf{N}$ & & -14.02737925 & 0.00107 & \\
\hline 70 & $\mathrm{M}$ & 148.554 & $\mathrm{~N}$ & & -14.06623952 & $-0.00033 *$ & \\
\hline 71 & $\mathbf{M}$ & 152.656 & W & & -14.37348998 & -0.00014 & \\
\hline 72 & $\mathrm{M}$ & 153.645 & $\mathbf{N}$ & & -14.41235025 & -0.00063 & \\
\hline 73 & $\mathbf{M}$ & 153.655 & $\mathbf{N}$ & & -14.41455666 & -0.00278 & \\
\hline 74 & $\mathbf{M}$ & 154.656 & $\mathrm{~N}$ & & -14.45562726 & $0.00015 *$ & \\
\hline 75 & $\mathbf{M}$ & 155.435 & $\mathbf{N}$ & & -14.48299745 & $0.00017 *$ & \\
\hline 76 & $\mathbf{M}$ & 155.445 & $\mathrm{~N}$ & & -14.48520386 & -0.00197 & \\
\hline 77 & $\mathbf{M}$ & 155.455 & $N$ & & -14.48741027 & -0.01065 & \\
\hline 78 & $\mathbf{M}$ & 155.545 & 3 & & -14.48984569 & 0.00098 & \\
\hline 79 & $\mathbf{M}$ & 155.555 & 3 & & -14.49205210 & -0.00661 & \\
\hline 80 & $\mathrm{M}$ & 155.565 & 3 & & -14.49425851 & 0.00086 & \\
\hline 81 & $\mathrm{M}$ & 155.645 & $N$ & & -14.49448752 & 0.00085 & \\
\hline 82 & $\mathrm{M}$ & 155.655 & $\mathbf{N}$ & M1 & -14.49669393 & $-0.02964 *$ & $\mathrm{E} / \mathrm{K} 1 \mathrm{M}$ \\
\hline 83 & $\mathbf{M}$ & 155.665 & $N$ & & -14.49890034 & -0.00594 & \\
\hline 84 & $\mathbf{M}$ & 155.675 & $\mathrm{~N}$ & & -14.50110675 & 0.00017 & \\
\hline 85 & $\mathbf{M}$ & 156.555 & $\mathbf{N}$ & & -14.53312073 & $0.00016 *$ & \\
\hline 86 & $\mathbf{M}$ & 156.654 & $N$ & & -14.53776060 & $-0.00018 *$ & \\
\hline 87 & $\mathbf{M}$ & 157.445 & $N$ & & -14.56734113 & 0.00016 & \\
\hline 88 & $\mathbf{M}$ & 157.455 & $\mathrm{~N}$ & KI1 & -14.56954754 & $-0.00566 *$ & $\mathrm{EV} / \mathrm{K} 1 \mathrm{M}$ \\
\hline 89 & M & 157.465 & $\mathrm{~N}$ & & -14.57175395 & -0.00124 & \\
\hline 90 & $\mathbf{M}$ & 158.454 & $\mathrm{~N}$ & & -14.61061422 & $-0.00024 *$ & \\
\hline 91 & $\mathrm{~S}$ & 161.557 & $\mathrm{~N}$ & & -14.87679800 & 0.00042 & \\
\hline 92 & $\mathbf{S}$ & 162.556 & $N$ & PI1 & -14.91786469 & 0.01029 & $\mathrm{E} / \mathrm{P} 1$ \\
\hline
\end{tabular}


Table II (Continued)

\begin{tabular}{|c|c|c|c|c|c|c|c|}
\hline & & Argument & & & $\begin{array}{c}\text { Frequency } \\
\omega_{i}\end{array}$ & Amplitude & Remarks \\
\hline \multicolumn{5}{|c|}{ Resonance frequency J.V. } & -14.9387894 & & $\mathrm{R}$ model \\
\hline 93 & $\mathbf{M}$ & 163.535 & $\mathbf{N}$ & & -14.95451854 & $0.00014 *$ & \\
\hline 94 & $\mathrm{M}$ & 163.545 & $\mathbf{N}$ & & -14.95672495 & -0.00199 & \\
\hline 95 & SM & 163.555 & $\mathbf{N}$ & P1 & -14.95893136 & 0.17584 & \\
\hline 96 & $\mathrm{~s}$ & 163.557 & $\mathrm{~N}$ & & -14.95893528 & $-0.00011 *$ & \\
\hline 97 & $\mathrm{M}$ & 163.755 & $\mathrm{~N}$ & & -14.96821503 & $-0.00026 *$ & \\
\hline 98 & $S$ & 164.554 & $\mathbf{N}$ & & -14.99999804 & -0.00147 & \\
\hline 99 & $\mathrm{~s}$ & 164.556 & $\mathrm{~N}$ & S1 & -15.00000196 & $-0.00423 *$ & $\mathrm{E} / \mathrm{K} 1 \mathrm{~S}$ \\
\hline 100 & $\mathrm{M}$ & 165.455 & 3 & & -15.03642680 & -0.00036 & \\
\hline 101 & $\mathrm{M}$ & 165.545 & $\mathrm{~N}$ & & -15.03886222 & 0.01050 & \\
\hline 102 & MS & 165.555 & $\mathbf{P}$ & K1 & -15.04106863 & -0.53050 & Sidereal day \\
\hline 103 & $M$ & 165.565 & $N$ & & -15.04327504 & -0.07182 & \\
\hline 104 & $\mathrm{M}$ & 165.575 & $\mathrm{~N}$ & & -15.04548145 & 0.00154 & \\
\hline 105 & M & 165.655 & 3 & & -15.04571046 & -0.00013 & \\
\hline \multicolumn{5}{|c|}{ Resonance frequency mol. } & -15.0732651 & & Model 1 \\
\hline \multicolumn{5}{|c|}{ Resonance frequency mol. } & -15.0736125 & & Model 2 \\
\hline \multicolumn{5}{|c|}{ Resonance frequency } & -15.0747606 & & CP model \\
\hline 106 & $\mathbf{S}$ & 166.554 & $\mathrm{~N}$ & PSI1 & -15.08213530 & $-0.00423 *$ & $\mathrm{E} / \mathrm{K} 1 \mathrm{~S}$ \\
\hline \multicolumn{3}{|c|}{ Resonance frequency } & J.V. & & -15.1016841 & & $\mathrm{R}$ model \\
\hline 107 & $\mathrm{M}$ & 167.355 & $\mathrm{~N}$ & & -15.11392223 & $-0.00026 *$ & \\
\hline 108 & $\mathrm{~S}$ & 167.553 & $\mathrm{~N}$ & & -15.12320198 & $-0.00011 *$ & \\
\hline 109 & $\mathrm{~S}$ & 167.555 & $\mathrm{~N}$ & FI1 & -15.12320590 & -0.00756 & \\
\hline 110 & $\mathrm{M}$ & 167.565 & $\mathrm{~N}$ & & -15.12541231 & 0.00029 & \\
\hline 111 & $\mathbf{M}$ & 167.575 & $\mathbf{N}$ & & -15.12761872 & $0.00014 *$ & \\
\hline 112 & $\mathrm{~S}$ & 168.554 & $\mathrm{~N}$ & & -15.16427258 & -0.00044 & \\
\hline 113 & $\mathrm{M}$ & 172.656 & $\mathrm{~N}$ & & -15.47152304 & $-0.00024 *$ & \\
\hline 114 & $\mathrm{M}$ & 173.445 & $\mathbf{N}$ & & -15.50109965 & -0.00017 & \\
\hline 115 & $\mathrm{M}$ & 173.645 & $\mathrm{~N}$ & & -15.51038331 & 0.00018 & \\
\hline 116 & $\mathrm{M}$ & 173.655 & $\mathrm{~N}$ & TT1 & -15.51258972 & $-0.00566 *$ & $\mathrm{EV} / \mathrm{K} 1 \mathrm{M}$ \\
\hline 117 & $\mathbf{M}$ & 173.665 & $\mathbf{N}$ & & -15.51479613 & -0.00112 & \\
\hline 118 & $\mathrm{M}$ & 173.765 & 3 & & -15.51943797 & -0.00089 & \\
\hline 119 & $\mathrm{M}$ & 174.456 & $\mathrm{~N}$ & & -15.54437666 & $-0.00018 *$ & \\
\hline 120 & $\mathbf{M}$ & 174.555 & $\mathrm{~N}$ & & -15.54901653 & $0.00016 *$ & \\
\hline 121 & $\mathbf{M}$ & 175.445 & $\mathrm{~N}$ & & -15.58323692 & 0.00087 & \\
\hline 122 & $\mathrm{M}$ & 175.455 & $\mathrm{~N}$ & 31 & -15.58544333 & $-0.02964 *$ & $\mathrm{E} / \mathrm{K} 1 \mathrm{M}$ \\
\hline 123 & $\mathrm{M}$ & 175.465 & $\mathrm{~N}$ & & -15.58764974 & -0.00587 & \\
\hline 124 & M & 175.475 & W & & -15.58985615 & 0.00013 & \\
\hline 125 & $\mathrm{M}$ & 175.555 & 3 & & -15.59008516 & -0.00241 & \\
\hline 126 & $\mathrm{M}$ & 175.655 & $\mathrm{~N}$ & & -15.59472699 & 0.00046 & \\
\hline 127 & $\mathrm{M}$ & 175.665 & $\mathrm{~N}$ & & -15.59693340 & 0.00029 & \\
\hline 128 & $\mathbf{M}$ & 175.675 & $\mathrm{~N}$ & & -15.59913981 & $0.00017 *$ & \\
\hline 129 & $\mathrm{M}$ & 176.454 & $\mathrm{~N}$ & & -15.62651000 & $0.00015 *$ & \\
\hline 130 & $\mathbf{M}$ & 177.455 & $\mathrm{~N}$ & & -15.66758060 & 0.00012 & \\
\hline 131 & $\mathrm{M}$ & 182.556 & $\mathrm{~N}$ & & -16.01589774 & $-0.00032 *$ & \\
\hline 132 & $\mathrm{M}$ & 183.545 & $\mathbf{N}$ & & -16.05475801 & -0.00016 & \\
\hline 133 & $\mathbf{M}$ & 183.555 & $\mathrm{~N}$ & SO1 & -16.05696442 & $-0.00492 *$ & $\mathrm{~V} / \mathrm{K} 1 \mathrm{M}$ \\
\hline 134 & $\mathbf{M}$ & 183.565 & $\mathrm{~N}$ & & -16.05917083 & -0.00096 & \\
\hline 135 & $\mathrm{M}$ & 185.355 & $\mathrm{~N}$ & & -16.12981802 & $-0.00240 *$ & \\
\hline 136 & $\mathrm{M}$ & 185.365 & $\mathrm{~N}$ & & -16.13202443 & -0.00048 & \\
\hline 137 & $\mathrm{M}$ & 185.455 & 3 & & -16.13445986 & -0.00040 & \\
\hline 138 & $\mathrm{M}$ & 185.465 & 3 & & -16.13666627 & -0.00016 & \\
\hline
\end{tabular}


Table II (Continued)

\begin{tabular}{|c|c|c|c|c|c|c|c|}
\hline & & Argument & & & $\begin{array}{l}\text { Frequency } \\
\omega_{i}\end{array}$ & Amplitude & Remarks \\
\hline 139 & $\mathbf{M}$ & 185.555 & $\mathrm{~N}$ & OO1 & -16.13910169 & -0.01623 & \\
\hline 140 & M & 185.565 & $\mathbf{N}$ & & -16.14130810 & -0.01039 & \\
\hline 141 & $\mathbf{M}$ & 185.575 & $\mathrm{~N}$ & & -16.14351451 & $-0.00218 *$ & \\
\hline 142 & $\mathbf{M}$ & 185.585 & W & & -16.14572092 & -0.00014 & \\
\hline 143 & $\mathbf{M}$ & 191.655 & $w$ & & -16.52848550 & -0.00015 & \\
\hline 144 & M & 193.455 & $\mathrm{~N}$ & & -16.60133912 & $-0.00078 *$ & \\
\hline 145 & $\mathbf{M}$ & 193.465 & $\mathrm{~N}$ & & -16.60354553 & -0.00015 & \\
\hline 146 & $\mathrm{M}$ & 193.655 & $\mathrm{~N}$ & & -16.61062278 & -0.00059 & \\
\hline 147 & $\mathbf{M}$ & 193.665 & & & -16.61282919 & -0.00038 & \\
\hline 148 & $M$ & 195.255 & w & & -16.67419271 & $-0.00019 *$ & \\
\hline 149 & $\mathbf{M}$ & 195.455 & $\mathrm{~N}$ & NU1 & -16.68347639 & -0.00311 & $\mathrm{E} / \mathrm{OO} 1$ \\
\hline 150 & M & 195.465 & $N$ & & -16.68568280 & -0.00199 & \\
\hline 151 & M & 195.475 & $\mathbf{N}$ & & -16.68788921 & $-0.00042 *$ & \\
\hline 152 & $\mathbf{M}$ & $1 \times 3.555$ & $\mathrm{~N}$ & & -17.15499748 & -0.00050 & \\
\hline 153 & M & $1 \times 3.565$ & $\mathrm{~N}$ & & -17.15720389 & -0.00032 & \\
\hline 154 & $\mathrm{M}$ & $1 \times 5.355$ & $\mathbf{N}$ & & -17.22785108 & -0.00041 & \\
\hline 155 & $\mathbf{M}$ & $1 \times 5.365$ & $\mathrm{~N}$ & & -17.23005749 & -0.00027 & \\
\hline 156 & $\mathbf{M}$ & $1 \mathrm{E} 3.455$ & $N$ & & -17.69937218 & -0.00012 & \\
\hline
\end{tabular}

$(X=10, E=11$, if appearing in place of digits)

Remarks

E Elliptic

EV Evection

$\mathrm{V} \quad$ Variation

$\mathrm{R}$ model Roche's model

CP model Central particle model

sponding to $s, h, p, N, p_{s}$ ) and in Doodson's code-number. One can easily find the arguments of the two associated tides (written in Doodson's codified notation).

Arguments already represented in the Doodson's tidal table are marked with a point.

Negative argument nutations are indicated by an $X$.

It must be seen that a few pairs of corresponding tides have exactly the same amplitude: $A_{i}=-A_{-i}$. If so, from (1), $\Delta \theta$ is theoretically zero; we have noted such pairs by an $*$ to indicate that this zero is not an approximative one.

Table II. This table reproduces Doodson's diurnal tides development. Waves corresponding to a term appearing in the IAU nutation development are noted with an $\mathrm{N}$ and a few waves given in Woolard's development by a W.

Table III. This table gives in sidereal and mean solar days, the period of each nutation associated with a diurnal tesseral tidal wave. This period can easily be deduced from

$$
P=\frac{\omega}{\omega_{i}-\omega}=\frac{\omega}{\Delta \omega_{i}}
$$

(diurnal waves deduced from the third-order potential are eliminated). 


\begin{tabular}{|c|c|c|c|c|c|c|c|c|}
\hline & & Argumen & & & Amplitude & Frequency & Nutation & \\
\hline & & & & & & $\omega_{i}$ & $\overline{\text { Sid. day }}$ & Solar day \\
\hline 1 & $\mathbf{M}$ & 105.955 & & & 0.00011 & -11.76553679 & 4.591947 & 4.579409 \\
\hline 2 & M & 107.755 & & & 0.00046 & -11.83839039 & 4.696403 & 4.683580 \\
\hline 3 & $\mathrm{M}$ & 109.555 & & & 0.00028 & -11.91124400 & 4.805722 & 4.792600 \\
\hline 5 & $\mathbf{M}$ & 115.845 & & & 0.00021 & -12.30770508 & 5.502769 & 5.487744 \\
\hline 6 & M & 115.855 & $\mathrm{~N}$ & & 0.00108 & -12.30991149 & 5.507214 & 5.492177 \\
\hline 8 & M & 117.645 & & & 0.00053 & -12.38055867 & 5.653453 & 5.638016 \\
\hline 9 & M & 117.655 & $\mathbf{N}$ & & 0.00278 & -12.38276508 & 5.658145 & 5.642696 \\
\hline 10 & M & 118.654 & & & 0.00021 & -12.42383176 & 5.746926 & 5.731235 \\
\hline 11 & M & 119.445 & & & 0.00010 & -12.45341229 & 5.812622 & 5.796751 \\
\hline 12 & M & 119.455 & & & 0.00054 & -12.45561970 & 5.817584 & 5.801700 \\
\hline 13 & M & 124.756 & & & -0.00013 & -12.81321951 & 6.751385 & 6.732951 \\
\hline 16 & M & 125.745 & $\mathbf{N}$ & & 0.00180 & -12.85207977 & 6.871240 & 6.852478 \\
\hline 17 & M & 125.755 & $\mathrm{~N}$ & 2Q1 & 0.00955 & -12.85428618 & 6.878173 & 6.859392 \\
\hline 18 & M & 126.556 & & & -0.00016 & -12.88607310 & 6.979628 & 6.960571 \\
\hline 19 & $\mathrm{M}$ & 126.655 & & & -0.00011 & -12.89071297 & 6.994688 & 6.975590 \\
\hline 20 & $\mathrm{M}$ & 126.754 & & & 0.00015 & -12.89535285 & 7.009814 & 6.990674 \\
\hline 22 & $\mathrm{M}$ & 127.545 & $\mathrm{~N}$ & & 0.00218 & -12.92493337 & 7.107801 & 7.088393 \\
\hline 23 & $\mathbf{M}$ & 127.555 & $\mathrm{~N}$ & SIG1 & 0.01153 & -12.92713978 & 7.115219 & 7.095792 \\
\hline 24 & M & 128.544 & & & 0.00014 & -12.96600005 & 7.248468 & 7.228676 \\
\hline 25 & $\mathbf{M}$ & 128.554 & $\mathbf{N}$ & & 0.00079 & -12.96820646 & 7.256183 & 7.236371 \\
\hline 26 & $\mathrm{M}$ & 129.355 & W & & 0.00035 & -12.99999339 & 7.369188 & 7.349067 \\
\hline 27 & $\mathbf{M}$ & 133.855 & W & & -0.00023 & -13.32580728 & 8.768966 & 8.745023 \\
\hline 28 & $\mathrm{M}$ & 134.656 & $\mathbf{N}$ & & -0.00061 & -13.35759420 & 8.934539 & 8.910144 \\
\hline 29 & M & 135.435 & W & & -0.00028 & -13.38496439 & 9.082199 & 9.057400 \\
\hline 32 & $\mathrm{M}$ & 135.635 & $\mathrm{~N}$ & & $-0.00042 *$ & -13.39424805 & 9.133398 & 9.108460 \\
\hline 33 & $\mathbf{M}$ & 135.645 & $\mathrm{~N}$ & & 0.01360 & -13.39645446 & 9.145651 & 9.120680 \\
\hline 34 & M & 135.655 & $\mathrm{~N}$ & Q1 & 0.07216 & -13.39866087 & 9.157938 & 9.132932 \\
\hline 36 & & 135.855 & W & & $-0.00019 *$ & -13.40794455 & 9.209997 & 9.184850 \\
\hline 37 & $\mathrm{M}$ & 136.456 & & & -0.00013 & -13.43044780 & 9.338677 & 9.313178 \\
\hline 38 & $\mathbf{M}$ & 136.555 & W & & -0.00039 & -13.43508767 & 9.365658 & 9.340085 \\
\hline 39 & $\mathbf{M}$ & 136.644 & & & 0.00011 & -13.43752113 & 9.379870 & 9.354259 \\
\hline 40 & $\mathbf{M}$ & 136.654 & $\mathrm{~N}$ & & 0.00068 & -13.43972754 & 9.392795 & 9.367148 \\
\hline 41 & M & 137.445 & & & 0.00258 & -13.46930807 & 9.569567 & 9.543438 \\
\hline 42 & $\mathbf{M}$ & 137.455 & $\mathrm{~N}$ & RO1 & 0.01371 & -13.47151448 & 9.583019 & 9.556854 \\
\hline 44 & M & 137.655 & $\mathrm{~N}$ & & $-0.00078 *$ & -13.48079814 & 9.640039 & 9.613717 \\
\hline 45 & M & 137.665 & W & & 0.00024 & -13.48300455 & 9.653690 & 9.627331 \\
\hline 46 & $M$ & 138.444 & & & 0.00011 & -13.51037475 & 9.826307 & 9.799477 \\
\hline 47 & $\mathbf{M}$ & 138.454 & $\mathrm{~N}$ & & 0.00064 & -13.51258116 & 9.840491 & 9.813623 \\
\hline 48 & M & 139.455 & W & & -0.00014 & -13.55365176 & 10.112207 & 10.084597 \\
\hline 49 & M & 143.535 & W & & -0.00017 & -13.85648548 & 12.697351 & 12.662682 \\
\hline 50 & M & 143.745 & W & & -0.00020 & -13.86797556 & 12.821718 & 12.786709 \\
\hline 51 & M & 143.755 & $\mathrm{~N}$ & & -0.00113 & -13.87018179 & 12.845897 & 12.810804 \\
\hline 52 & $\mathbf{M}$ & 144.546 & W & & -0.00015 & -13.89976249 & 13.178820 & 13.142836 \\
\hline 53 & $\mathrm{M}$ & 144.556 & $\mathbf{N}$ & & -0.00130 & -13.90196890 & 13.204347 & 13.168293 \\
\hline 55 & M & 145.535 & $\mathbf{N}$ & & $-0.00218 *$ & -13.93862275 & 13.643362 & 13.606110 \\
\hline 56 & M & 145.545 & $\mathrm{~N}$ & & 0.07105 & -13.94082916 & 13.670722 & 13.633395 \\
\hline 57 & M & 145.555 & & O1 & 0.37689 & -13.94303557 & 13.698192 & 13.660790 \\
\hline 61 & M & 145.755 & $\mathrm{~N}$ & & $-0.00243 *$ & -13.95231924 & 13.814996 & 13.777275 \\
\hline 62 & $\mathbf{M}$ & 145.765 & $\mathrm{~N}$ & & -0.00040 & -13.95452565 & 13.843049 & 13.805252 \\
\hline 63 & $\mathrm{M}$ & 146.544 & W & & 0.00012 & -13.98189583 & 14.200769 & 14.161995 \\
\hline
\end{tabular}




\begin{tabular}{|c|c|c|c|c|c|c|c|c|}
\hline & & \multirow[t]{2}{*}{ Argument } & & & \multirow[t]{2}{*}{ Amplitude } & \multirow{2}{*}{$\begin{array}{l}\text { Frequency } \\
\qquad \omega_{i}\end{array}$} & \multicolumn{2}{|c|}{ Nutation Period } \\
\hline & & & & & & & Sid. day & Solar day \\
\hline 64 & M & 146.554 & $N$ & & 0.00115 & -13.98410224 & 14.230413 & 14.191558 \\
\hline 65 & $\mathbf{M}$ & 147.355 & W & & $-0.00491 *$ & -14.01588917 & 14.671644 & 14.631584 \\
\hline 67 & $\mathbf{M}$ & 147.545 & $N$ & & -0.00021 & -14.02296643 & 14.773633 & 14.733294 \\
\hline 68 & $\mathrm{M}$ & 147.555 & $\mathbf{N}$ & TO1 & 0.00014 & -14.02517284 & 14.805720 & 14.765293 \\
\hline 69 & $\mathrm{M}$ & 147.565 & $N$ & & 0.00107 & -14.02737925 & 14.837946 & 14.797432 \\
\hline 70 & $\mathrm{M}$ & 148.554 & $\mathrm{~N}$ & & $-0.00033 *$ & -14.06623952 & 15.429441 & 15.387312 \\
\hline 71 & $\mathrm{M}$ & 152.656 & $\mathbf{W}$ & & -0.00014 & -14.37349898 & 22.530781 & 22.469262 \\
\hline 72 & $\mathrm{M}$ & 153.645 & $\mathbf{N}$ & & -0.00063 & -14.41235025 & 23.923379 & 23.858058 \\
\hline 73 & M & 153.655 & $N$ & & -0.00278 & -14.41455666 & 24.007631 & 23.942080 \\
\hline 74 & $M$ & 154.656 & $\mathbf{N}$ & & $0.00015 *$ & -14.45562726 & 25.691844 & 25.621694 \\
\hline 75 & M & 155.435 & $\mathrm{~N}$ & & $0.00017 *$ & -14.48299745 & 26.951882 & 26.878291 \\
\hline 76 & $M$ & 155.445 & $\mathbf{N}$ & & -0.00197 & -14.48520386 & 27.058862 & 26.984980 \\
\hline 77 & $\mathbf{M}$ & 155.455 & $N$ & & -0.01065 & -14.48741027 & 27.166696 & 27.092519 \\
\hline 81 & $\mathrm{M}$ & 155.645 & $\mathrm{~N}$ & & 0.00085 & -14.49448752 & 27.518456 & 27.443319 \\
\hline 82 & M & 155.655 & $N$ & M1 & $-0.02964 *$ & -14.49669393 & 27.629992 & 27.554550 \\
\hline 83 & M & 155.665 & $\mathrm{~N}$ & & -0.00594 & -14.49890034 & 27.742435 & 27.666686 \\
\hline 84 & M & 155.675 & $\mathrm{~N}$ & & 0.00017 & -14.50110675 & 27.855797 & 27.779738 \\
\hline 85 & $\mathrm{M}$ & 156.555 & $\mathbf{N}$ & & $0.00016 *$ & -14.53312073 & 29.611439 & 29.530587 \\
\hline 86 & $\mathrm{M}$ & 156.654 & $\mathrm{~N}$ & & $-0.00018 *$ & -14.53776060 & 29.884420 & 29.802822 \\
\hline 87 & $\mathrm{M}$ & 157.445 & $\mathrm{~N}$ & & 0.00016 & -14.56734113 & 31.750465 & 31.663772 \\
\hline 88 & $\mathbf{M}$ & 157.455 & $\mathbf{N}$ & KIl & $-0.00566 *$ & -14.56954754 & 31.899036 & 31.811938 \\
\hline 89 & $\mathrm{M}$ & 157.465 & $N$ & & -0.00124 & -14.57175395 & 32.049005 & 31.961497 \\
\hline 90 & $\mathrm{M}$ & 158.454 & $\mathbf{N}$ & & $-0.00024 *$ & -14.61061422 & 34.942303 & 34.846895 \\
\hline 91 & $\mathrm{~S}$ & 161.557 & $\mathrm{~N}$ & & 0.00042 & -14.87679800 & 91.562737 & 91.312731 \\
\hline 92 & $\mathrm{~S}$ & 162.556 & $N$ & PIl & 0.01029 & -14.91786469 & 122.082691 & 121.749353 \\
\hline 93 & $\mathrm{M}$ & 163.535 & $\mathrm{~N}$ & & $0.00014 *$ & -14.95451854 & 173.784552 & 173.310044 \\
\hline 94 & $\mathbf{M}$ & 163.545 & $\mathrm{~N}$ & & -0.00199 & -14.95672495 & 178.330713 & 177.843793 \\
\hline 95 & SM & 163.555 & $\mathrm{~N}$ & Pl & 0.17584 & -14.95893136 & 183.121117 & 182.621116 \\
\hline 96 & $\mathbf{S}$ & 163.557 & $\mathbf{N}$ & & $-0.00011 *$ & -14.95893528 & 183.129856 & 182.629832 \\
\hline 97 & $\mathrm{M}$ & 163.755 & $\mathrm{~N}$ & & $-0.00026 *$ & -14.96821503 & 206.456079 & 205.892364 \\
\hline 98 & $\mathrm{~S}$ & 164.554 & $N$ & & -0.00147 & -14.99999804 & 366.224800 & 365.224848 \\
\hline 99 & $\mathrm{~S}$ & 164.556 & $\mathrm{~N}$ & S1 & $-0.00423 *$ & -15.00000196 & 366.259758 & 365.259710 \\
\hline 101 & $\mathrm{M}$ & 165.545 & $\mathrm{~N}$ & & 0.01050 & -15.03886222 & 6816.987155 & 6798.373824 \\
\hline 102 & MS & 165.555 & $\mathbf{P}$ & KI & -0.53050 & -15.04106863 & $\infty$ & $\infty$ \\
\hline 103 & $\mathrm{M}$ & 165.565 & $N$ & & -0.07182 & -15.04327504 & -6816.987155 & -6798.373824 \\
\hline 104 & $\mathrm{M}$ & 165.575 & $\mathrm{~N}$ & & 0.00154 & -15.04548145 & -3408.493577 & -3399.186912 \\
\hline 106 & $S$ & 166.554 & $\mathbf{N}$ & PSIl & $-0.00423 *$ & -15.08213530 & -366.259758 & -365.259710 \\
\hline 107 & $\mathrm{M}$ & 167.355 & $N$ & & $-0.00026 *$ & -15.11392223 & -206.456079 & -205.892364 \\
\hline 108 & $\mathrm{~S}$ & 167.553 & $\mathrm{~N}$ & & $-0.00011 *$ & -15.12320198 & -183.129856 & -182.629832 \\
\hline 109 & $\mathrm{~S}$ & 167.555 & $\mathrm{~N}$ & FI1 & -0.00756 & -15.12320590 & -183.121117 & -182.621116 \\
\hline 110 & $\mathrm{M}$ & 167.565 & $N$ & & 0.00029 & -15.12541231 & -178.330713 & -177.843793 \\
\hline 111 & $\mathrm{M}$ & 167.575 & $\mathrm{~N}$ & & $0.00014 *$ & -15.12761872 & -173.784552 & -173.310044 \\
\hline 112 & $\mathrm{~S}$ & 168.554 & $\mathbf{N}$ & & -0.00044 & -15.16427258 & -122.082681 & -121.749343 \\
\hline 113 & $\mathrm{M}$ & 172.656 & $\mathrm{~N}$ & & $-0.00024 *$ & -15.47152304 & -34.942303 & -34.846895 \\
\hline 114 & $\mathbf{M}$ & 173.445 & $\mathrm{~N}$ & & -0.00017 & -15.50109965 & -32.695770 & -32.606496 \\
\hline 115 & $\mathbf{M}$ & 173.645 & $\mathrm{~N}$ & & 0.00018 & -15.51038331 & -32.049005 & -31.961497 \\
\hline 116 & $\mathrm{M}$ & 173.655 & $\mathrm{~N}$ & TT1 & $-0.00566 *$ & -15.51258972 & -31.899036 & -31.811938 \\
\hline 117 & $\mathbf{M}$ & 173.665 & $\mathrm{~N}$ & & -0.00111 & -15.51479613 & -31.750465 & -31.663772 \\
\hline 119 & $\mathrm{M}$ & 174.456 & $N$ & & $-0.00018 *$ & -15.54437666 & -29.884420 & -29.802822 \\
\hline 120 & $\mathbf{M}$ & 174.555 & $N$ & & $0.00016 *$ & -15.54901653 & -29.611439 & -29.530587 \\
\hline
\end{tabular}


Table III (Continued)

\begin{tabular}{|c|c|c|c|c|c|c|c|c|}
\hline & \multirow{2}{*}{\multicolumn{2}{|c|}{ Argument }} & & & \multirow[t]{2}{*}{ Amplitude } & \multirow{2}{*}{$\begin{array}{l}\text { Frequency } \\
\quad \omega_{i}\end{array}$} & \multicolumn{2}{|c|}{ Nutation Period } \\
\hline & & & & & & & Sid. day & Solar day \\
\hline 121 & M & 175.445 & $\mathrm{~N}$ & & 0.00087 & -15.58323692 & -27.742435 & -27.666686 \\
\hline 122 & M & 175.455 & $\mathrm{~N}$ & J1 & $-0.02964 *$ & -15.58544333 & -27.629992 & -27.554550 \\
\hline 123 & M & 175.465 & $\mathrm{~N}$ & & -0.00587 & -15.58764974 & -27.518456 & -27.443319 \\
\hline 124 & M & 175.475 & W & & 0.00013 & -15.58985615 & -27.407818 & -27.332983 \\
\hline 126 & M & 175.655 & $\mathrm{~N}$ & & 0.00046 & -15.59472699 & -27.166696 & -27.092519 \\
\hline 127 & M & 175.665 & $\mathbf{N}$ & & 0.00029 & -15.59693340 & -27.058862 & -26.984980 \\
\hline 128 & M & 175.675 & $\mathbf{N}$ & & $0.00017 *$ & -15.59913981 & -26.951882 & -26.878291 \\
\hline 129 & M & 176.454 & $\mathrm{~N}$ & & $0.00015 *$ & -15.62651000 & -25.691844 & -25.621694 \\
\hline 130 & M & 177.455 & $\mathbf{N}$ & & 0.00012 & -15.66758060 & -24.007631 & -23.942080 \\
\hline 131 & $\mathrm{M}$ & 182.556 & $\mathrm{~N}$ & & $-0.00032 *$ & -16.01589774 & -15.429441 & -15.387312 \\
\hline 132 & $\mathrm{M}$ & 183.545 & $\mathrm{~N}$ & & -0.00016 & -16.05475801 & -14.837946 & -14.797432 \\
\hline 133 & M & 183.555 & $\mathrm{~N}$ & SO1 & $-0.00492 *$ & -16.05696442 & -14.805720 & -14.765293 \\
\hline 134 & $\mathrm{M}$ & 183.565 & $\mathrm{~N}$ & & -0.00096 & -16.05917083 & -14.773633 & -14.733294 \\
\hline 135 & $\mathbf{M}$ & 185.355 & $\mathbf{N}$ & & $-0.00240 *$ & -16.12981802 & -13.814996 & -13.777275 \\
\hline 136 & M & 185.365 & $\mathrm{~N}$ & & -0.00048 & -16.13202443 & -13.787055 & -13.749411 \\
\hline 139 & M & 185.555 & $\mathrm{~N}$ & OO1 & -0.01623 & -16.13910169 & -13.698192 & -13.660790 \\
\hline 140 & M & 185.565 & $\mathbf{N}$ & & -0.01039 & -16.14130810 & -13.670722 & -13.633395 \\
\hline 141 & M & 185.575 & $\mathbf{N}$ & & $-0.00218 *$ & -16.14351451 & -13.643362 & -13.606110 \\
\hline 142 & M & 185.585 & W & & -0.00014 & -16.14572092 & -13.616111 & -13.578933 \\
\hline 143 & M & 191.655 & W & & -0.00015 & -16.52848550 & -10.112207 & -10.084597 \\
\hline 144 & M & 193.455 & $\mathrm{~N}$ & & $-0.00078 *$ & -16.60133912 & -9.640039 & -9.613717 \\
\hline 145 & M & 193.465 & $\mathbf{N}$ & & -0.00015 & -16.60354553 & -9.626426 & -9.600141 \\
\hline 146 & M & 193.655 & $\mathbf{N}$ & & -0.00059 & -16.61062278 & -9.583019 & -9.556854 \\
\hline 147 & M & 193.665 & & & -0.00038 & -16.61282919 & -9.569567 & -9.543438 \\
\hline 148 & M & 195.255 & W & & $-0.00019 *$ & -16.67419271 & -9.209997 & -9.184850 \\
\hline 149 & $\mathrm{M}$ & 195.455 & $\mathrm{~N}$ & NU1 & -0.00311 & -16.68347639 & -9.157938 & -9.132932 \\
\hline 150 & $\mathbf{M}$ & 195.465 & $\mathbf{N}$ & & -0.00199 & -16.68568280 & -9.145651 & -9.120680 \\
\hline 151 & M & 195.475 & $\mathbf{N}$ & & $-0.00042 *$ & -16.68788921 & -9.133398 & -9.108460 \\
\hline 152 & & $1 \times 3.555$ & $\mathrm{~N}$ & & -0.00050 & -17.15499748 & -7.115219 & -7.095792 \\
\hline 153 & & $1 \times 3.565$ & $\mathrm{~N}$ & & -0.00032 & -17.15720389 & -7.107801 & -7.088393 \\
\hline 154 & & $1 \times 5.355$ & $\mathbf{N}$ & & -0.00041 & -17.22785108 & -6.878173 & -6.859392 \\
\hline 155 & & $1 \times 5.365$ & $\mathrm{~N}$ & & -0.00027 & -17.23005749 & -6.871240 & -6.852478 \\
\hline 156 & & $1 \mathrm{E} 3.455$ & $\mathrm{~N}$ & & -0.00012 & -17.69937218 & -5.658145 & -5.642696 \\
\hline
\end{tabular}

$(\mathrm{X}=10, \mathrm{E}=11$, if appearing in place of digits)

\section{Calculation of Nutation Amplitudes from the Static Tidal Development}

Equations (1) permit a very simple calculation of these amplitudes, the coefficient $E_{\mathbb{R}}$ must be determined from the precession constant $(C-A) / C$.

Here appears the incoherence of our system of fundamental constants. It is wellknown that adopting $1 / 81.30$ for the relative Moon's mass and the adopted IAU value for precession, one finds for the nutation constant

$$
N=9.222 \text { (cf. Table IV) }
$$

while IAU admits

$$
N=9^{\prime \prime} 2100 \text {. }
$$


If one uses the proposed value 50 ". 40 for the precession constant, one will find

$$
N=9.2272, \text { (cf. Table IV) }
$$

the discrepancy being emphasized.

We have calculated two models of nutation tables from the tidal tables in taking respectively

$$
\begin{array}{llll}
E_{\mathbb{q}}=0.0164120 & \text { giving } & N=9^{\prime \prime} .2100 & \text { (Table VA) } \\
E_{\downarrow}=0.0164427 & \text { giving } & N=9.2272 & \text { (Table VB) }
\end{array}
$$

Comparing Tables VA and I, one ascertains a small disagreement in the semi-annual solar nutation 57.555 and a small divergency in the ellipticity of principal nutation 55.565. All the other waves are perfectly identical. We shall explain later these two small discrepancies.

\section{Comparison Between Nutation Tables and Observations. Ellipticity of the Principal Nutation}

(a) Table VI presents first a set of 'theoretical' coefficients following several authors. One can notice a certain number of internal contradictions due to the fact that these authors have adopted different values of fundamental constants (precession, Moon's mass).

TABLE IV

\begin{tabular}{llll}
\hline$P$ & \multicolumn{1}{l}{$\mu^{-1}$} & $N$ & $K$ \\
\hline 50.37000 & 81.3000 & 9.221661 & 9.221858 \\
50.37500 & 81.3000 & 9.222576 & 9.222774 \\
50.38000 & 81.3000 & 9.223492 & 9.223689 \\
50.38500 & 81.3000 & 9.224407 & 9.224604 \\
50.39000 & 81.3000 & 9.225322 & 9.225520 \\
50.39500 & 81.3000 & 9.226238 & 9.226435 \\
50.40000 & 81.3000 & 9.227153 & 9.227351 \\
50.40500 & 81.3000 & 9.228069 & 9.228266 \\
& & & \\
50.40000 & 81.2900 & 9.227508 & 9.227705 \\
50.40000 & 81.2950 & 9.227331 & 9.227528 \\
50.40000 & 81.3000 & 9.227153 & 9.227351 \\
50.40000 & 81.3050 & 9.226976 & 9.227173 \\
50.40000 & 81.3100 & 9.226798 & 9.226996 \\
50.40000 & 81.3150 & 9.226621 & 9.226819 \\
50.40000 & 81.3200 & 9.226444 & 9.226641 \\
50.40000 & 81.3250 & 9.226266 & 9.226464 \\
50.40000 & 81.3300 & 9.226089 & 9.226287 \\
50.40000 & 81.3350 & 9.225912 & 9.226109 \\
50.40000 & 81.3400 & 9.225734 & 9.225932 \\
& & & \\
$N$ & \multicolumn{3}{l}{ Newcomb's formula } \\
$K$ & Kulikov's formula & \\
& & &
\end{tabular}


TABLE VA

$E_{\mathrm{Q}}=0.0164120$

\begin{tabular}{|c|c|c|c|c|c|c|c|c|}
\hline & & & Arg. & Tides & Period & $\sin \theta \cdot \Delta \psi$ & $\Delta \theta$ & Nut.arg \\
\hline 9 & 156 & M & 117.655 & $1 \mathrm{E} 3.455$ & 5.658145 & -0.000247 & 0.000269 & $\mathrm{X} 3.455$ \\
\hline 16 & 155 & $\mathbf{M}$ & 125.745 & $1 \times 5.365$ & 6.871240 & -0.000172 & 0.000233 & 95.365 \\
\hline 17 & 154 & M & 125.755 & $1 \times 5.355$ & 6.878173 & -0.001031 & 0.001124 & 95.355 \\
\hline 22 & 153 & $M$ & 127.545 & $1 \times 3.565$ & 7.107801 & -0.000216 & 0.000291 & 93.565 \\
\hline 23 & 152 & $\mathrm{M}$ & 127.555 & $1 \times 3.555$ & 7.115219 & -0.001288 & 0.001404 & 93.555 \\
\hline 32 & 151 & M & 135.635 & 195.475 & 9.133398 & 0.000125 & 0.000000 & 85.475 \\
\hline 33 & 150 & $\mathrm{M}$ & 135.645 & 195.465 & 9.145651 & -0.001742 & 0.002340 & 85.465 \\
\hline 34 & 149 & M & 135.655 & 195.455 & 9.157938 & -0.010378 & 0.011313 & 85.455 \\
\hline 36 & 148 & M & 135.855 & 195.255 & 9.209997 & 0.000057 & 0.000000 & 85.255 \\
\hline 41 & 147 & $\mathrm{M}$ & 137.445 & 193.665 & 9.569567 & -0 & 0.000464 & 3.665 \\
\hline 42 & 146 & $\mathrm{M}$ & 137.455 & 193.655 & 9.583019 & -0.002063 & 0.002249 & 3.655 \\
\hline 44 & 144 & $\mathbf{M}$ & 137.655 & 193.455 & 9.640039 & 0.000246 & 0.000000 & 83.455 \\
\hline 48 & 143 & $\mathrm{M}$ & 139.455 & 191.655 & 10.112207 & 0.000048 & 0.000001 & 81.655 \\
\hline 55 & 141 & M & 145.535 & 75 & 362 & 76 & 0.000000 & .575 \\
\hline 56 & 140 & $\mathrm{M}$ & 145.545 & 185.565 & 13.670722 & -0.013609 & 0.018272 & 75.565 \\
\hline 57 & 139 & M & 145.555 & 185.555 & 13.698192 & -0.081081 & 0.088379 & 75.555 \\
\hline 61 & 135 & $\mathrm{M}$ & 145.755 & 185.355 & 13.814996 & 0.001095 & -0.000006 & 75.355 \\
\hline 67 & 134 & M & 147.545 & 183.565 & 14.773633 & 0.000198 & 0.000266 & 73.565 \\
\hline 68 & 133 & $\mathbf{M}$ & 147.555 & 183.555 & 14.805720 & 0.002388 & 0.000002 & 73.555 \\
\hline 69 & 132 & M & 147.565 & 15 & 46 & -0.1 & 0.00 & 73.545 \\
\hline 70 & 131 & $\mathrm{M}$ & 148.554 & 182.556 & 15.429441 & 0.000164 & -0.000002 & 72.556 \\
\hline 73 & 130 & $\mathrm{M}$ & 153.655 & 177.455 & 24.007631 & 0.001048 & -0.001142 & 67.455 \\
\hline 74 & 129 & M & 154.656 & 176.454 & 25.691844 & -0.000126 & 0.000000 & 66.454 \\
\hline 75 & 128 & M & 155.435 & 75 & 26.951882 & -0 & 0.00 & 575 \\
\hline 76 & 127 & $\mathrm{M}$ & 155.445 & 175.665 & 27.0 & 0.000746 & -0.001003 & 5.665 \\
\hline 77 & 126 & M & 155.455 & 175.655 & 27.166696 & 0.004543 & -0.004953 & 65.655 \\
\hline 81 & 123 & M & 155.645 & 175.465 & 27.518456 & 0.002267 & 0.003034 & 65.465 \\
\hline 82 & 122 & $\mathrm{M}$ & 155.655 & 175.455 & 27.629992 & 0.026881 & 0.000000 & 65.455 \\
\hline 83 & 121 & M & 155.665 & 175.445 & 27.742435 & 0.002308 & -0.003100 & 65.445 \\
\hline 85 & 120 & M & 156.555 & 55 & 29.6 & $-0 . \mathrm{C}$ & 0.00 & 555 \\
\hline 86 & 119 & M & & & & & & 4.456 \\
\hline 87 & 117 & M & 157.445 & 173.665 & 31.750465 & 0.000500 & 0.000666 & 63.665 \\
\hline 88 & 116 & $\mathrm{M}$ & 157.455 & 173.655 & 31.899036 & 0.005926 & 0.000000 & 63.655 \\
\hline 89 & 115 & $\mathrm{M}$ & 157.465 & 173.645 & 32.049005 & 0.000557 & -0.000746 & 63.645 \\
\hline 90 & 113 & $\mathrm{M}$ & 158.454 & 172.656 & 34.942303 & 0.000275 & 0.000000 & 62.656 \\
\hline 92 & 112 & $\mathrm{~S}$ & 162.556 & 168.554 & 122.082681 & -0.019735 & 498 & 58.554 \\
\hline 93 & 111 & $\mathbf{M}$ & 163.535 & 167.575 & 173.784552 & -0.000798 & 0.000000 & 57.575 \\
\hline 94 & 110 & $\mathrm{M}$ & 163.545 & 167.565 & 178.330713 & 0.004975 & -0.006673 & 57.565 \\
\hline 95 & 109 & SM & 163.555 & 167.555 & 183.121117 & -0.505745 & 0.551187 & 57.555 \\
\hline 96 & 108 & $\mathbf{S}$ & 163.557 & 167.553 & 183.129856 & 0.000661 & 0.000000 & 57.553 \\
\hline 97 & 107 & M & 163.755 & 167.355 & 206.456079 & 0.001761 & & 57.355 \\
\hline 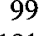 & 10 & $\mathrm{~S}$ & & & & 853 & 0.000000 & 56.554 \\
\hline 101 & 103 & M & 165.545 & 165.565 & 6816.987155 & 6.860505 & 9.209993 & 55.565 \\
\hline
\end{tabular}

$(X=10, E=11$, if appearing in place of digits $)$ 
TABLE VB

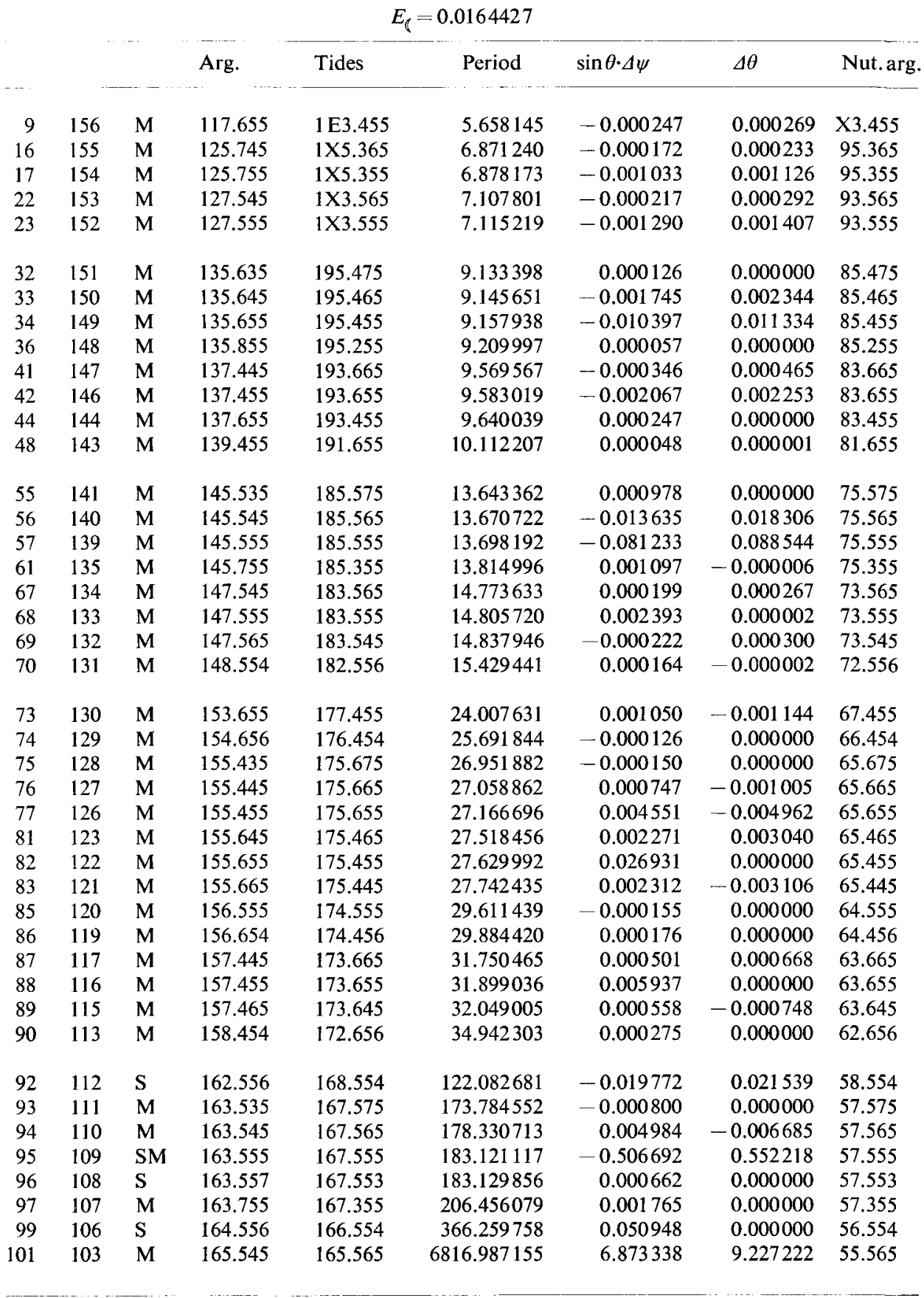

$(X=10, E=11$, if appearing in place of digits) 
TABLE VI

Principal Nutations Amplitudes

(1) Theory

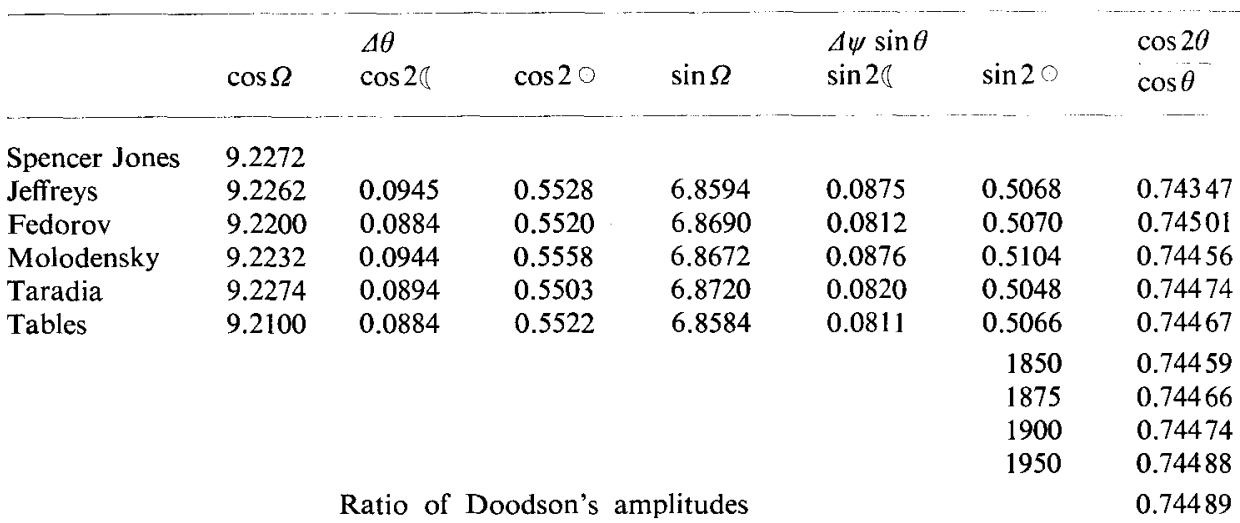

(2) Theory

Liquid core models

$\begin{array}{llllllll} & & & & & & & \\ \text { Jeffr. Vic. R } & 9.2187 & 0.0971 & 0.5403 & 6.8491 & 0.0897 & 0.4883 & 0.74296 \\ \text { Jeffr. Vic. CP } & 9.2015 & 0.0972 & 0.5734 & 6.8260 & 0.0896 & 0.5232 & 0.74184 \\ \text { Molodensky 1 } & 9.1963 & 0.0969 & 0.5770 & 6.8325 & 0.0899 & 0.5274 & 0.74296 \\ \text { Molodensky 2 } & 9.1997 & 0.0965 & 0.5745 & 6.8369 & 0.0895 & 0.5255 & 0.74317\end{array}$

Observations

$\begin{array}{llllllll}\text { Przybyllok } & 9.2069 & & & & & & \\ \text { Spencer Jones } & 9.2066 & & & & & & \\ \text { Morgan } & 9.206 & 0.0980 & & & & & \\ \text { Clemence } & 9.2070 & & & & & & \\ \text { Fedorov-Jeffr. } & 9.1980 & 0.0949 & 0.5780 & 6.8530 & 0.0918 & 0.5330 & 0.74505 \\ \text { Fedorov 1967 } & 9.1974 & 0.0965 & 0.578 & 6.8437 & 0.0934 & 0.533 & 0.74510 \\ \begin{array}{l}\text { Evtouchenko } \\ \text { Taradia }\end{array} & 9.1970 & 0.099 & & & & & 0.74455 \\ \text { Popov } & & & 0.578 & 6.8476 & & 0.533 & \\ \text { Wako A } & & & 0.5673 & & & 0.5209 & \\ \text { Wako B } & & & 0.5716 & & & 0.5252 & \end{array}$

Moreover, the ratio of the principal nutation ellipse axes is fairly variable. This ratio whose value from the static theory is

$$
\frac{\cos 2 \theta}{\cos \theta}
$$

corresponds to $\theta(1875)$ in the IAU nutation table and to $\theta(1950)$ in the tidal tables (Doodson says he has adopted the 1900 value but it seems that this small discrepancy is finally due to truncation errors in the calculation). That is the reason why in Table VA one finds 6.8605 instead of 6".8584 (IAU) for the ellipse semi-minor axis. 
(b) Observations give a systematically weaker $N$ value:

$$
N \sim 9.20
$$

while other significant disagreements appear for the semi-annual and semi-monthly nutations.

These discrepancies are attributed to dynamical effects of the Earth's liquid core.

\section{Dynamical Effects of the Earth's Liquid Core}

Tesseral forces of diurnal tides generate the precession-nutation torque which tends to rotate the equator towards the ecliptic.

Once applied to the liquid core, this torque tends to create core motions with respect to the Earth's mantle in which our reference axes are fixed.

The hydrodynamical theory (Poincaré) shows the existence of resonance frequencies. They were calculated for a few models firstly by Jeffreys and Vicente (1957) and by Molodensky (1961) afterwards.

These frequencies are indicated in Table II. They are very near to the solar wave $\psi_{1}$ (166.554) corresponding to the annual nutation 56.554 .

The central line of the tidal spectrum is the 165.555 one $\left(K_{1}\right)$ corresponding to a sidereal day; pairs of symmetric lines with respect to this central one generate nutations.

The displacement of the resonance line with respect to the central one produces a dissymmetric distortion on the $A_{i}$ amplitudes; then the effect of resonance is different for $A_{i}$ and $A_{-i}$.

If so, the nutation ellipse axes $\left(A_{i}+A_{-i}\right),\left(A_{i}-A_{-i}\right)$ are modified in different proportions and their ratio is no longer $\cos 2 \theta / \cos \theta$ as can be seen in the liquid core models list of Table VI. One will never have rigorously

$$
A_{i}=A_{-i}
$$

and never nutations purely in longitude. The most important case will be evidently the annual nutation one 56.554 which because of the near proximity of the resonance line $\left(\psi_{1}=166.554\right)$ will have a non-negligible obliquity component.

\section{Experimental Proofs of Dynamical Effects of the Liquid Core by Earth Tides Measurements}

Earth tide measurements have been considerably developed since 1959. Using continuous-recording apparatus, it has been possible to obtain observational series of 1000 to 2000 days, the analysis of which was conclusive for the problem considered here.

In tidal phenomena, principal waves (of maximum amplitudes) are not the same as those in nutation, because of the factor $\omega / \Delta \omega_{i}$ in Equations (1). 
One can actually isolate the following waves with certitude:
$\begin{array}{ll}K_{1} & 165.555\end{array}$
associated with
55.555
precession
$P_{1} \quad 163.555$
associated with
53.555
semi-annual nutation
$O_{1} \quad 145.555$
associated with
75.555
semi-monthly nutation
$Q_{1} \quad 135.655$
associated with
85.455 9-day nutation

and one searches for

$$
\psi_{1} \quad 166.554 \text { associated with } 56.554 \text { annual nutation. }
$$

It is known that the used parameters in Earth tides interpretation, as in the Chandlerian polar motion, are Love's numbers $h, k$ and $l$ (Melchior, 1966).

Performed measurements are interpreted in amplitude ratio form (observed to theoretical amplitude); they give linear combinations of Love's numbers:

$$
\begin{array}{ll}
\text { one for the horizontal component } & \gamma=1+k-h \\
\text { one for the vertical component } & \delta=1+h-\frac{3}{2} k \\
\text { one for the fundamental astronomy } & A=1+k-l
\end{array}
$$

Table VII presents results of the liquid core resonance effect for all principal waves. Calculations were made for two Earth models following Molodensky's theory:

$$
\begin{array}{ll}
\text { resonance factor } & \beta \\
\text { Love's number } & h \text { and } k \\
\text { combinations } & \gamma \text { and } \delta
\end{array}
$$

Waves at the two tide spectrum extremities $\left(Q_{1}, O_{1}, O O_{1}, v_{1}\right)$ are little affected by resonance effect and closely correspond to the purely static theory. Figure 1 illustrates these results.

Experimental results are summarized in Table VIII. They are based on more than 50000 days of tide recordings in the best conditions. Special attention has been drawn to the problem of instrumental calibration.

For the vertical component (North American and Askania), recording gravimeters calibrated on traditional gravimetric bases are used.

For horizontal components, quartz horizontal pendulums Verbaandert-Melchior are provided, which are supplied with an automatic calibration system related by interferometric measurements to the green line of mercury.

The resonance effect is most striking in horizontal components for

$$
k \approx \frac{1}{2} h
$$

and then

$$
\gamma \approx 1-\frac{h}{2} \quad \delta \approx 1+\frac{h}{4} .
$$

Horizontal pendulum measurements are thus more favorable in the study of dynamical effects of the core. 
TABLE VII

Molodensky's Theory

\begin{tabular}{|c|c|c|c|c|c|c|c|}
\hline & & A & Frequency $\omega_{i}$ & $\Delta \omega_{i}=\omega_{i}-\omega$ & $\omega / \Delta \omega_{i}$ & $\begin{array}{l}\beta \\
\text { Mod. } 1\end{array}$ & $\begin{array}{l}\beta \\
\text { Mod. } 2\end{array}$ \\
\hline Q1 & 135.655 & 0.07216 & -13.39866087 & 1.64240777 & 9.157938 & 5.26 & 5.00 \\
\hline O1 & 145.555 & 0.37689 & -13.94303557 & 1.09803307 & 13.698193 & 7.08 & 6.50 \\
\hline M1 & 155.655 & -0.02964 & -14.49669393 & 0.54437471 & 27.629992 & 12.45 & 12.06 \\
\hline PI1 & 162.556 & 0.01029 & -14.91786469 & 0.12320395 & 122.082681 & 42.23 & 41.20 \\
\hline P1 & 163.555 & 0.17554 & -14.95893136 & 0.08213728 & 183.121117 & 56.86 & 55.50 \\
\hline \multirow[t]{2}{*}{ S1 } & 164.556 & -0.00423 & -15.00000196 & 0.04106668 & 366.259758 & 87.89 & 85.73 \\
\hline & 165.545 & 0.01050 & -15.03886223 & 0.00220641 & 6816.987155 & 185.30 & 180.17 \\
\hline \multirow[t]{3}{*}{ K1 } & 165.555 & -0.53050 & -15.04106864 & 0.00000000 & $\infty$ & 197.92 & 192.30 \\
\hline & 165.565 & -0.07182 & -15.04327505 & -0.00220641 & -6816.987155 & 212.41 & 206.20 \\
\hline & 165.575 & 0.00154 & -15.04548147 & -0.00441283 & -3408.485856 & 229.21 & 222.94 \\
\hline PS1 & 166.554 & -0.00423 & -15.08213530 & -0.04106666 & -366.259758 & 717.52 & 728.04 \\
\hline \multirow[t]{2}{*}{ FII } & 167.555 & -0.00756 & -15.12320590 & -0.08213726 & -183.121117 & -125.17 & -124.06 \\
\hline & 168.554 & -0.00044 & -15.16427259 & -0.12320395 & -122.082681 & -61.91 & -61.22 \\
\hline Jl & 175.455 & -0.02964 & -15.58544333 & -0.54437469 & -27.629992 & -10.87 & -10.86 \\
\hline OOI & 185.555 & -0.01623 & -16.13910169 & -1.09803305 & -13.698193 & -4.45 & -4.54 \\
\hline NUI & 195.455 & -0.00311 & -16.68347639 & -1.64240775 & -9.157938 & -2.44 & -2.57 \\
\hline
\end{tabular}

\begin{tabular}{|c|c|c|c|c|c|c|c|c|}
\hline \multicolumn{5}{|c|}{ Model 1} & \multicolumn{4}{|c|}{ Model 2} \\
\hline & $h$ & $k$ & $\gamma$ & $\delta$ & $h$ & $k$ & $\gamma$ & $\delta$ \\
\hline Q1 & 0.621 & 0.307 & 0.686 & 1.160 & 0.615 & 0.300 & 0.685 & 1.165 \\
\hline $\mathrm{O} 1$ & 0.618 & 0.305 & 0.687 & 1.160 & 0.614 & 0.300 & 0.686 & 1.166 \\
\hline PII & 0.601 & 0.297 & 0.696 & 1.155 & 0.599 & 0.292 & 0.693 & 1.161 \\
\hline P1 & 0.594 & 0.294 & 0.700 & 1.153 & 0.593 & 0.288 & 0.695 & 1.161 \\
\hline \multirow[t]{2}{*}{ S1 } & 0.579 & 0.286 & 0.707 & 1.150 & 0.580 & 0.281 & 0.701 & 1.158 \\
\hline & 0.533 & 0.263 & 0.730 & 1.138 & 0.540 & 0.259 & 0.719 & 1.151 \\
\hline \multirow[t]{2}{*}{$\mathrm{K} 1$} & 0.527 & 0.260 & 0.733 & 1.137 & 0.535 & 0.256 & 0.721 & 1.151 \\
\hline & 0.521 & 0.256 & 0.735 & 1.137 & 0.529 & 0.252 & 0.723 & 1.151 \\
\hline PSI1 & 0.959 & 0.478 & 0.520 & 1.242 & 0.928 & 0.475 & 0.547 & 1.215 \\
\hline FI1 & 0.680 & 0.337 & 0.657 & 1.174 & 0.670 & 0.331 & 0.661 & 1.173 \\
\hline$J 1$ & 0.626 & 0.310 & 0.684 & 1.161 & 0.621 & 0.304 & 0.683 & 1.165 \\
\hline OO1 & 0.623 & 0.308 & 0.685 & 1.161 & 0.619 & 0.303 & 0.684 & 1.165 \\
\hline Stat. & 0.621 & 0.307 & 0.686 & 1.160 & & & & \\
\hline \multirow{2}{*}{\multicolumn{2}{|c|}{ Argument }} & \multirow{2}{*}{\multicolumn{2}{|c|}{$\begin{array}{l}\text { Nutation Period } \\
\text { M. Sid. day }\end{array}$}} & & \multicolumn{2}{|c|}{ Molodensky } & \multicolumn{2}{|c|}{$n / n_{0}$ Jeffreys } \\
\hline & & & & M. Sol. day & Mod. 1 & Mod. 2 & Mod. 1 & Mod. 2 \\
\hline Q1 & 135.655 & 9.15 & & 9.132933 & 1.0137 & 1.0121 & & \\
\hline $\mathrm{O} 1$ & 145.555 & 13.6 & & 13.660791 & 1.0254 & 1.0214 & 1.0269 & 1.0266 \\
\hline M1 & 155.655 & 27.6 & & 27.554550 & 1.0369 & 1.0353 & & \\
\hline PI1 & 162.556 & 122.08 & & 121.749353 & 1.0389 & 1.0372 & & \\
\hline$P 1$ & 163.555 & 183.12 & & 182.621117 & 1.0359 & 1.0344 & 1.0350 & 0.9707 \\
\hline \multirow[t]{2}{*}{$\mathrm{S} 1$} & 164.556 & 366.2 & & 365.259710 & 1.0286 & 1.0273 & & \\
\hline & 165.545 & 6816.98 & & 6798.373824 & 1.0037 & 1.0032 & 1.0036 & 1.0012 \\
\hline \multirow[t]{3}{*}{$\mathrm{K} 1$} & 165.555 & $\infty$ & & $\infty$ & & & & \\
\hline & 165.565 & -6816.98 & & -6798.373824 & 0.9962 & 0.9964 & 0.9964 & 0.9989 \\
\hline & 165.575 & -3408.48 & & -3399.179212 & 0.9918 & 0.9921 & & \\
\hline PSI & 166.554 & -366.2 & & -365.259710 & 1.2472 & 1.2448 & & \\
\hline \multirow[t]{2}{*}{ FI1 } & 167.555 & -183.12 & & -182.621117 & 1.0884 & 1.0857 & 1.0895 & 1.1420 \\
\hline & 168.554 & -122.08 & & -121.749353 & 1.0678 & 1.0814 & & \\
\hline $\mathbf{J} 1$ & 175.455 & -27.62 & & -27.554550 & 1.0687 & 1.0663 & & \\
\hline OO1 & 185.555 & -13.6 & & -13.660791 & 1.0798 & 1.0772 & 1.0768 & 1.0670 \\
\hline NUI & 195.455 & -9.15 & & -9.132933 & 1.0924 & 1.0897 & & \\
\hline
\end{tabular}




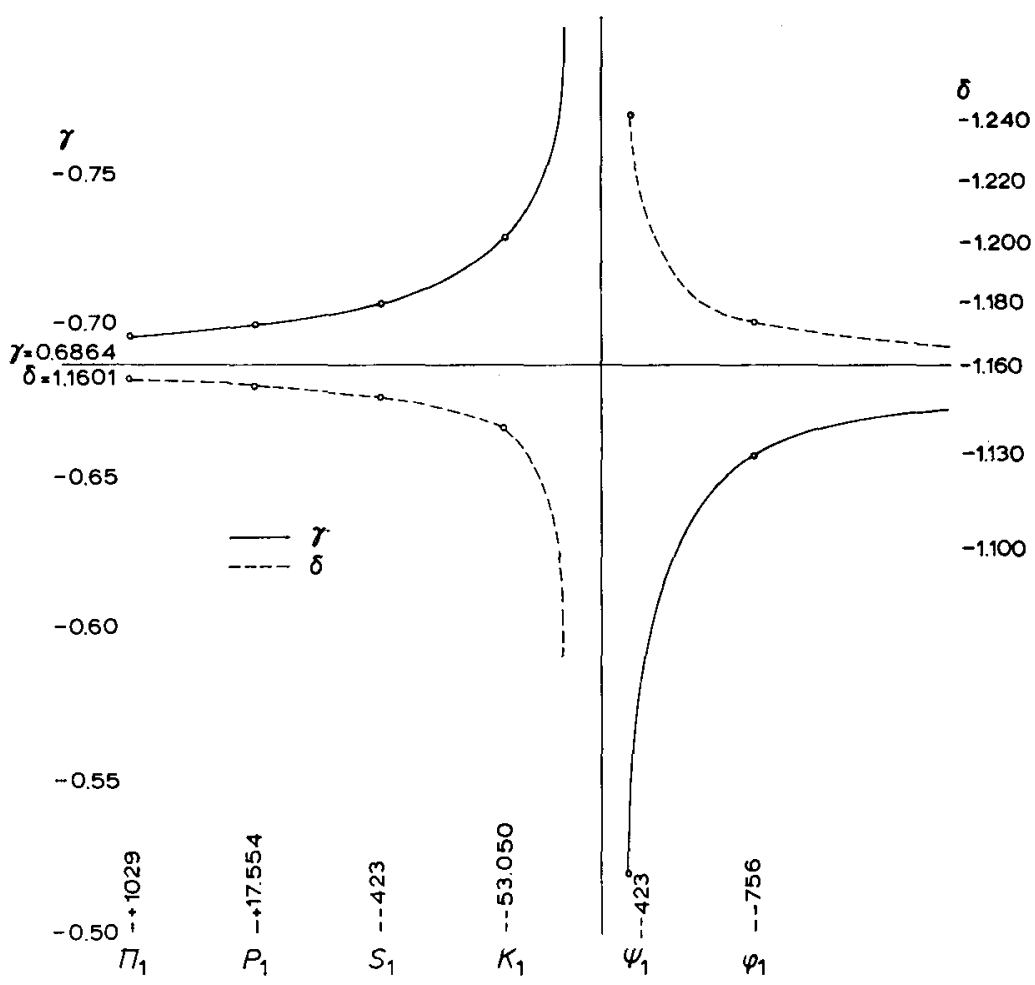

Fig. 1.

TABLE VIII

\begin{tabular}{lllll}
\hline Earth tides & & & \multicolumn{2}{c}{ Experimental results } \\
\hline Waves & $\begin{array}{l}\text { Factor } \\
\text { A.M. }\end{array}$ & $\begin{array}{l}\gamma=1+k-h \\
\text { W.M. }\end{array}$ & $\begin{array}{l}\text { Factor } \\
\text { A.M. }\end{array}$ & $\begin{array}{l}\delta=1+h-\frac{3}{2} k \\
\text { W.M. }\end{array}$ \\
\hline & & & & \\
K1 & 0.7422 & 0.7501 & 1.1406 & 1.1507 \\
P1 & 0.7068 & 0.7167 & 1.1699 & 1.1664 \\
O1 & 0.6785 & 0.6752 & 1.1522 & 1.1676 \\
Q1 & 0.6519 & 0.6374 & 1.1573 & 1.1684
\end{tabular}

Stations:

Sclaigneaux Walferdange Graz

Dourbes
Pribram
Dannemora
14.104 days
Arithmetic mean

\section{Kanne}

Tiefenort

Lohja

Uccle
Frankfurt
Trieste
Talgar

Luxembourg

Bonn

Resina

Kyoto

13.811 days
Strasbourg

Genova

Stockholm

Austin
Arithmetic mean
W.M. Weighted mean
A.M. 
Results published in Table VIII incontrovertibly carry one of the neatest confirmations of theoretical model calculations.

The wave $\psi_{1}$ which is most perturbed by resonance unluckily has a very weak amplitude. Four long observational series, by the best instruments (to our knowledge) - 2 gravimeters and 2 horizontal pendulums - confirm the predicted coefficients for models but the mean quadratic error is so considerable that results lose their meaning.

\section{Resonance Effect on Nutations}

The third part of Table VII gives the amplificational factor $n / n_{0}$ for each circular nutation associated with the principal tidal waves.

One restores elliptic nutations by recombination of two circular nutations with the same period, but of opposite sense.

Such a calculation was carried out in Tables IXA and IXB according to the two Molodensky's models; in each case, two calculations were executed corresponding to the following values:

$$
\begin{aligned}
& E_{\mathrm{Q}}=0.0164120 \text { and } E_{\mathbb{8}}=0.0164427 \\
& N_{0}=9.2100 \quad N_{0}=9.2272
\end{aligned}
$$

In the most interesting case,

$$
E_{\curlyvee}=0.0164427
$$

corresponding to

$$
\begin{aligned}
P & =50.400 \\
\mu^{-1} & =81.30
\end{aligned}
$$

one verifies that, in each model, the nutation constant is brought back to the value

$$
N=9.2014
$$

which is perfectly consistent with observational results.

In longitude, one nevertheless finds an amplitude of 6."840 a little weaker than the deduced observational value.

One remarks elsewhere in Table VI that the axes ratio, sensibly changed in liquid core models, does not seem altered in experimental results.

For the short-period nutations, we note the following points:

(a) semi-annual nutation

New amplitudes $(0.572,0$. 523$)$ agree very well with experimental results. Alteration with respect to the statical amplitudes $(0.552,0.507)$ is important.

(b) semi-monthly nutation

Jeffreys-Vicente and Molodensky have applied the resonance coefficient to already 
TABLE IXA

Resonance Effect on Nutations - Molodensky's Model 1

\begin{tabular}{|c|c|c|c|c|c|c|c|}
\hline & & Tidal arg. & & & Frequency & Amplitude & Period \\
\hline 34 & M & 135.655 & $\mathrm{~N}$ & Q1 & -13.39866087 & 0.07315 & 9.157938 \\
\hline 149 & $\mathbf{M}$ & 195.455 & $\mathrm{~N}$ & NU1 & -16.68347639 & -0.00340 & 9.157938 \\
\hline 57 & $\mathbf{M}$ & 145.555 & $\mathbf{N}$ & $\mathrm{O} 1$ & -13.94303557 & 0.38646 & 13.698192 \\
\hline 139 & M & 185.555 & $\mathrm{~N}$ & OO1 & -16.13910169 & -0.01753 & 13.698192 \\
\hline 82 & $\mathrm{M}$ & 155.655 & $\mathrm{~N}$ & M1 & -14.49669393 & -0.03073 & 27.629992 \\
\hline 122 & $\mathrm{M}$ & 175.455 & $\mathbf{N}$ & $\mathrm{J} 1$ & -15.58544333 & -0.03168 & 27.629992 \\
\hline 112 & $\mathrm{~S}$ & 168.554 & $\mathrm{~N}$ & & -15.16427258 & -0.00047 & 122.082681 \\
\hline 92 & $S$ & 162.556 & $\mathrm{~N}$ & PI1 & -14.91786469 & 0.01069 & 122.082681 \\
\hline 95 & SM & 163.555 & $\mathbf{N}$ & P1 & -14.95893136 & 0.18215 & 183.121117 \\
\hline 109 & $\mathbf{S}$ & 167.555 & $\mathrm{~N}$ & FI1 & -15.12320590 & -0.00823 & 183.121117 \\
\hline 99 & $\mathrm{~S}$ & 164.556 & $\mathrm{~N}$ & S1 & -15.00000196 & -0.00435 & 366.259758 \\
\hline 106 & $\mathbf{S}$ & 166.554 & $\mathbf{N}$ & PSI1 & -15.08213530 & -0.00528 & 366.259758 \\
\hline 101 & $\mathbf{M}$ & 165.545 & $\mathrm{~N}$ & & -15.03886222 & 0.01054 & 6816.987155 \\
\hline 103 & $\mathbf{M}$ & 165.565 & $\mathbf{N}$ & & -15.04327504 & -0.07155 & 6816.987155 \\
\hline
\end{tabular}

$E_{\mathbb{8}}=0.0164120 \quad$ Rigid Earth 9.2100

\begin{tabular}{|c|c|c|c|c|c|c|c|c|}
\hline & & \multicolumn{3}{|c|}{ Tidal arg. } & \multirow{2}{*}{$\begin{array}{l}\text { Period } \\
9.157938\end{array}$} & \multirow{2}{*}{$\frac{\sin \theta \cdot \Delta \psi}{-0.010483}$} & \multirow{2}{*}{$\begin{array}{c}\Delta \theta \\
0.011505\end{array}$} & \multirow{2}{*}{$\begin{array}{l}\text { Arg. } \\
85.455\end{array}$} \\
\hline 34 & 149 & $\mathbf{M}$ & 135.655 & 195.455 & & & & \\
\hline 57 & 139 & $\mathbf{M}$ & 145.555 & 185.555 & 13.698192 & -0.082940 & 0.090822 & 75.555 \\
\hline 82 & 122 & M & 155.655 & 175.455 & 27.629992 & 0.028300 & 0.000430 & 65.455 \\
\hline 112 & 92 & $\mathrm{~S}$ & 168.554 & 162.556 & 122.082681 & -0.020477 & -0.022360 & 58.554 \\
\hline 95 & 109 & SM & 163.555 & 167.555 & 183.121117 & -0.522696 & 0.572164 & 57.555 \\
\hline 99 & 106 & $\mathrm{~S}$ & 164.556 & 166.554 & 366.259758 & 0.057886 & 0.005590 & 56.554 \\
\hline 101 & 103 & M & 165.545 & 165.565 & 6816.987155 & 6.825822 & 9.184261 & 55.565 \\
\hline \multicolumn{3}{|c|}{$E_{\mathbb{8}}=0.0164427$} & \multicolumn{2}{|c|}{ Rigid Earth 9.2272} & & & & \\
\hline 34 & 149 & $\mathbf{M}$ & 135.655 & 195.455 & 9.157938 & -0.010503 & 0.011526 & 85.455 \\
\hline 57 & 139 & M & 145.555 & 185.555 & 13.698192 & -0.083096 & 0.090992 & 75.555 \\
\hline 82 & 122 & $\mathbf{M}$ & 155.655 & 175.455 & 27.629992 & 0.028353 & 0.000431 & 65.455 \\
\hline 112 & 92 & $\mathrm{~S}$ & 168.554 & 162.556 & 122.082681 & -0.020515 & -0.022402 & 58.554 \\
\hline 95 & 109 & SM & 163.555 & 167.555 & 183.121117 & -0.523674 & 0.573235 & 57.555 \\
\hline 99 & 106 & $S$ & 164.556 & 166.554 & 366.259758 & 0.057994 & 0.005600 & 56.554 \\
\hline 101 & 103 & M & 165.545 & 165.565 & 6816.987155 & 6.838591 & 9.201441 & 55.565 \\
\hline
\end{tabular}

modified amplitudes (0.0945 and 0.0875 in stead of 0.0884 and 0.0811$)$; this explains the higher coefficients obtained by them with respect to Tables IXA, IXB $(0.0970$ and 0.0897 against 0.0910 and 0.0831 ).

\section{(c) annual nutation}

This nutation associated with $\psi_{1}$ and $S_{1}$ waves lies nearest to resonance. Its amplitude is thus strongly modified: from $(0.0502,0.0000)$ to $(0.0579,0.0056)$. We must therefore introduce an annual nutation in obliquity. 
TABLE IXB

Resonance Effect on Nutations - Molodensky's Model 2

\begin{tabular}{|c|c|c|c|c|c|c|c|}
\hline & & Tidal arg. & & & Frequency & Amplitude & Period \\
\hline 34 & M & 135.655 & $\mathrm{~N}$ & Q1 & -13.39866087 & 0.07303 & 9.157938 \\
\hline 149 & M & 195.455 & $\mathrm{~N}$ & NU1 & -16.68347639 & -0.00339 & 9.157938 \\
\hline 57 & M & 145.555 & $\mathbf{N}$ & $\mathrm{O} 1$ & -13.94303557 & 0.38496 & 13.698192 \\
\hline 139 & $\mathbf{M}$ & 185.555 & $\mathrm{~N}$ & 001 & -16.13910169 & -0.01748 & 13.698192 \\
\hline 82 & $\mathrm{M}$ & 155.655 & $\mathrm{~N}$ & M1 & -14.49669393 & -0.03069 & 27.629992 \\
\hline 122 & $M$ & 175.455 & $\mathrm{~N}$ & $\mathrm{~J}_{1}$ & -15.58544333 & -0.03161 & 27.629992 \\
\hline 112 & $\mathrm{~S}$ & 168.554 & $\mathrm{~N}$ & & -15.16427258 & -0.00048 & 122.082681 \\
\hline 92 & $\mathrm{~S}$ & 162.556 & $\mathrm{~N}$ & PII & -14.91786469 & 0.01067 & 122.082681 \\
\hline 95 & SM & 163.555 & $\mathbf{N}$ & P1 & -14.95893136 & 0.18189 & 183.121117 \\
\hline 109 & $\mathrm{~S}$ & 167.555 & $\mathrm{~N}$ & FI1 & -15.12320590 & -0.00821 & 183.121117 \\
\hline 99 & $\mathrm{~S}$ & 164.556 & $\mathrm{~N}$ & S1 & -15.00000196 & -0.00434 & 366.259758 \\
\hline 106 & $\mathrm{~S}$ & 166.554 & $\mathrm{~N}$ & PSI1 & -15.08213530 & -0.00527 & 366.259758 \\
\hline 101 & $\mathrm{M}$ & 165.545 & $\mathrm{~N}$ & & -15.03886222 & 0.01053 & 6816.987155 \\
\hline 103 & $M$ & 165.565 & $N$ & & -15.04327504 & -0.07156 & 6816.987155 \\
\hline
\end{tabular}

$E_{\mathbb{8}}=0.0164120 \quad$ Rigid Earth 9.2100

\begin{tabular}{|c|c|c|c|c|c|c|c|c|}
\hline & & & Tidal ar & & Period & $\sin \theta \cdot \Delta \psi$ & $\Delta \theta$ & Arg. \\
\hline 34 & 149 & $\mathrm{M}$ & 135.655 & 195.455 & 9.157938 & -0.010466 & 0.011485 & 85.455 \\
\hline 57 & 139 & $\mathbf{M}$ & 145.555 & 185.555 & 13.698192 & -0.082614 & 0.090474 & 75.555 \\
\hline 82 & 122 & $\mathrm{M}$ & 155.655 & 175.455 & 27.629992 & 0.028250 & 0.000417 & 65.455 \\
\hline 112 & 92 & $\mathrm{~S}$ & 168.554 & 162.556 & 122.082681 & -0.020416 & -0.022340 & 58.554 \\
\hline 95 & 109 & SM & 163.555 & 167.555 & 183.121117 & -0.521975 & 0.571323 & 57.555 \\
\hline 99 & 106 & $\mathrm{~S}$ & 164.556 & 166.554 & 366.259758 & 0.057766 & 0.005590 & 56.554 \\
\hline 101 & 103 & $\mathbf{M}$ & 165.545 & 165.565 & 6816.987155 & 6.828060 & 9.184261 & 55.565 \\
\hline
\end{tabular}

$E_{\mathbb{Q}}=0.0164427 \quad$ Rigid Earth 9.2272

$\begin{array}{rrlrrrrrr}34 & 149 & \text { M } & 135.655 & 195.455 & 9.157938 & -0.010486 & 0.011507 & 85.455 \\ 57 & 139 & \text { M } & 145.555 & 185.555 & 13.698192 & -0.082769 & 0.090643 & 75.555 \\ 82 & 122 & \text { M } & 155.655 & 175.455 & 27.629992 & 0.028303 & 0.000417 & 65.455 \\ 112 & 92 & \text { S } & 168.554 & 162.556 & 122.082681 & -0.020455 & -0.022382 & 58.554 \\ 95 & 109 & \text { SM } & 163.555 & 167.555 & 183.121117 & -0.522951 & 0.572392 & 57.555 \\ 99 & 106 & \text { S } & 164.556 & 166.554 & 366.259758 & 0.057874 & 0.005600 & 56.554 \\ 101 & 103 & \text { M } & 165.545 & 165.565 & 6816.987155 & 6.840832 & 9.201441 & 55.565\end{array}$

\section{Conclusions: Answers to Questions Proposed in the Preliminary Programme for the Heidelberg Colloquium}

SPECIFY THE THEORETICAL RELATIONSHIPS BETWEEN THE PRECESSIONAL CONSTANTS AND OTHER ASTRONOMICAL OR GEOPHYSICAL CONSTANTS

The $H \equiv(C-A) / C$ value deduced from the constant of precession and the Moon's mass does not permit us to construct an accurate table of the nutations.

It is necessary to introduce the resonance parameter $\beta$, expressing the dynamical effects of the Earth's liquid core. 
It is also necessary to adopt an ordered and systematic nutation classification corresponding to the tidal one in order to assure an easy comparison with geophysical phenomena.

ARE THE CURRENT THEORY AND ADOPTED CONSTANTS OF NUTATION OF ADEQUATE ACCURACY?

No. Indeed the effects of the liquid core considerably alter the principal nutations coefficients, especially the annual nutation one.

One term of annual nutation in obliquity must be added to the development. WHEN IS AN IMPROVED BASIS FOR NUTATION LIKELY TO BE AVAILABLE?

We estimate that the tesseral Earth tides measurements associated with nutations will provide necessary experimental confirmation of theories on the dynamical effects of the Earth's liquid core.

WHAT ARE THE PROS AND CONS OF ADOPTING AN IMPROVED BASIS FOR NUTATION?

Pros: (1) For a very long time, the nutation constant 9".2100 has been unsatisfactory and the desire to modify it is already an old one. The value 9".2014 would be satisfactory from theoretical and experimental points of view.

(2) It is recommended to modify the semi-annual and semi-monthly nutation coefficients in the sense of an increase because such a modification still satisfies theory and experience. This will permit us to reduce the residuals in time and latitude observations whose spectral analysis reveals the presence of the corresponding periods.

(3) It is necessary to modify the annual nutation in obliquity. This will reduce also the residuals of annual period.

\section{References}

Fedorov, E. P.: 1959, Astron. J. 64, 81-4.

Fedorov, E. P.: 1963, Nutation and Forced Motion of the Earth's Pole, Pergamon Press, Oxford. Jeffreys, H.: 1948, Monthly Notices Roy. Astron. Soc. 108, 206-9.

Jeffreys, H.: 1949, 1950, Monthly Notices Roy. Astron. Soc. 109, 670-87; 110, 460-6.

Jeffreys, H.: 1959a, Monthly Notices Roy. Astron. Soc. 119, 75-80.

Jeffreys, H.: 1959b, Astron. J. 64, 84-6.

Jeffreys, H. and Vicente, R. O.: 1957, Monthly Notices Roy. Astron. Soc. 117, 142-73.

Melchior, P.: 1966, The Earth Tides, Pergamon Press, Oxford.

Melchior, P. and Georis, B.: 1968, Phys. Earth Planet Interiors, Vol. 1, 267-87.

Molodensky, M. S.: 1961, Obs. Royal Belgique Commun. No. 188, 25-56.

Poincaré, H.: 1910, Bull. Astron. 27, 321-56.

Taradia, V. K.: 1965, Astron. Zhurn. Akad. Nauk SSSR 43, 1.

Taradia, V. K.: 1969, (in Russian), Astrometriya i Astrofiz., Kiev No. 2, 7-26.

Vicente, R. O.: 1965, Bull. Astron. 25, 109-113.

Wako, Y.: 1967, Proc. Intern. Latitude Obs. Mizusawa 7, 165-86. 